\title{
Fiber-Optic Temperature and Pressure Sensors Applied to Radiofrequency Thermal Ablation in Liver Phantom: Methodology and Experimental Measurements
}

\author{
Daniele Tosi, ${ }^{1,2}$ Edoardo Gino Macchi, ${ }^{3}$ and Alfredo Cigada ${ }^{4}$ \\ ${ }^{1}$ School of Engineering, Nazarbayev University, 53 Kabanbay Batyr, 010000 Astana, Kazakhstan \\ ${ }^{2}$ Optical Fibre Sensors Research Centre (OFSRC), University of Limerick, Limerick, Ireland \\ ${ }^{3}$ Dipartimento di Ingegneria Civile ed Architettura, Università di Pavia, Via Ferrata 3, 27100 Pavia, Italy \\ ${ }^{4}$ Dipartimento di Meccanica, Politecnico di Milano, Via La Masa 34, 20158 Milano, Italy \\ Correspondence should be addressed to Daniele Tosi; daniele.tosi@ul.ie
}

Received 14 October 2014; Accepted 15 December 2014

Academic Editor: Geoffrey A. Cranch

Copyright (C) 2015 Daniele Tosi et al. This is an open access article distributed under the Creative Commons Attribution License, which permits unrestricted use, distribution, and reproduction in any medium, provided the original work is properly cited.

\begin{abstract}
Radiofrequency thermal ablation (RFA) is a procedure aimed at interventional cancer care and is applied to the treatment of small- and midsize tumors in lung, kidney, liver, and other tissues. RFA generates a selective high-temperature field in the tissue; temperature values and their persistency are directly related to the mortality rate of tumor cells. Temperature measurement in up to 3-5 points, using electrical thermocouples, belongs to the present clinical practice of RFA and is the foundation of a physical model of the ablation process. Fiber-optic sensors allow extending the detection of biophysical parameters to a vast plurality of sensing points, using miniature and noninvasive technologies that do not alter the RFA pattern. This work addresses the methodology for optical measurement of temperature distribution and pressure using four different fiber-optic technologies: fiber Bragg gratings (FBGs), linearly chirped FBGs (LCFBGs), Rayleigh scattering-based distributed temperature system (DTS), and extrinsic FabryPerot interferometry (EFPI). For each instrument, methodology for ex vivo sensing, as well as experimental results, is reported, leading to the application of fiber-optic technologies in vivo. The possibility of using a fiber-optic sensor network, in conjunction with a suitable ablation device, can enable smart ablation procedure whereas ablation parameters are dynamically changed.
\end{abstract}

\section{Introduction}

Radiofrequency thermal ablation (RFA) is an emerging medical procedure for targeted treatment of tumors [16]. Pioneered through the ' 90 s by Rossi et al. $[7,8]$, RFA makes use of electrical irradiance through a miniature applicator percutaneously inserted at the point of treatment. Tumor cells are damaged by high-temperature conditions, and cytotoxicity is function of the local temperature value and its persistence [9]; $52^{\circ} \mathrm{C}$ for $60 \mathrm{~s}$ exposure is considered the clinical reference condition for cancer cells mortality, while over $60^{\circ} \mathrm{C}$ cells death is nearly instantaneous. Thermal dosimetry $[8,9]$ defines the temperature dose that is related to each mortality rate of tumor cells.

RFA makes use of RF irradiance to provide a selective heating, confined within the tumor $[1,8,10]$. A midpower (5-50 W) RF source operating at $350-500 \mathrm{kHz}$ is used to power a miniature ablation device (1-4 mm diameter), percutaneously inserted in the tumor and with an electrical active region of up to $1 \mathrm{~cm}$ length; ablation devices having stem, multitiled, or other shapes have been reported [11, 12]. By powering the ablator with RF, the electrical irradiance propagates in the biological tissue by conduction. RFA generates steep spatial $\left(3-5^{\circ} \mathrm{C} / \mathrm{mm}\right)$ and temporal $\left(0.4^{\circ} \mathrm{C} / \mathrm{s}\right)$ gradients that ensure that the treatment is confined to the tumor.

The outcome of RFA depends on the evolution of the electrical impedance throughout the ablation. In most tissues, the impedance at ambient temperature is $20-200 \Omega$ and slightly reduces during the first part of ablation. Whereas temperature at the ablation point reaches $95-100^{\circ} \mathrm{C}$, the water constituents of the tissues reach the ebullience point; the impedance of vapor, at RF, is orders of magnitude higher than that in liquid water; hence, after reaching the ablation peak, the target tissue is progressively insulated from the electrically active area, 
substantially neutralizing the irradiance. This phenomenon is extremely significant in hepatic tumors $[8,9,13-16]$, as liver is a soft tissue with high water concentration. When RFA is applied to liver tumors, the vapor-to-liquid transition causes an instantaneous rise of the tissue impedance that abruptly interrupts the ablation procedure: this effect limits RFA to small hepatic tumors (up to $3 \mathrm{~cm}$ in size), while the treatment of mid-to-large tumors via percutaneous RFA is subject to disruptive improvements of the whole ablation setup.

The RFA procedure can be substantially improved by using sensors operating at the point of treatment. Currently, temperature measurement in RFA is performed with miniature thermocouples (TCs) $[15,21,22]$. TCs allow measurement in up to 3-5 points within the tumor volume and can be either installed on the ablator or externally inserted. However, TCs are invasive and can perceivably alter the ablation pattern, suffer from hysteresis, and are limited to few sensing points. Alternatively to TCs, noncontact techniques such as infrared thermal imaging have been reported [23, 24], but they are limited to surface imaging with a direct line of sight.

Optical fiber sensors (OFS) [25] can significantly improve thermal measurement in RFA. OFS are smaller than their electrical counterparts, so as not to alter the ablation pattern and exhibit hysteresis. The use of distributed or quasidistributed sensing techniques allows unmatched density of sensing points per units of length, providing sensing below $0.1 \mathrm{~mm}$ spatial resolution. The application of fiberoptic thermal sensors has been limited to few preliminary studies in $\mathrm{RF}[17,18,26]$ and laser $[27,28]$ ablation. Using OFS, it is also possible to increase the number of sensing points per unit of volume, by using inline wavelength division multiplexing (WDM) approaches and progressing towards quasidistributed and distributed sensing.

In this paper, methodologies for temperature detection in RFA are detailed, progressing from a fiber Bragg grating (FBG) array (1 sensing point $/ \mathrm{cm}$ ) to a linearly chirped FBG (LCFBG) for quasidistributed measurement (130 sensing points $/ \mathrm{cm}$ ) to a distributed temperature sensing system based on Rayleigh backscattering (100-500 sensing points $/ \mathrm{cm}$ ). Experimental measurements have been carried out ex vivo on liver phantom; several chambers have been developed in order to confine the tissue and position the fiber along different ablation axes. In addition to temperature measurement, the pressure readout is a key asset to support the advanced treatment of encapsulated tumors [29]. This measurement is addressed with a Fabry-Perot fiber-optic sensor [18, 30], with a low thermal coefficient, and is combined with a fiber Bragg grating sensor.

Biophysical sensing applied to RFA allows monitoring this treatment with unprecedented precision in real time. When RFA is applied to small ablations, for example, for thermal treatment of small tumors in hard tissues $[1,2]$, in correction of cardiac arrhythmia [31] or for interventional pain management, it is possible to adequately control the ablation procedure by just timing the RF irradiance. However, when RFA is applied to the treatment of midlarge tumors, particularly in soft tissues, the ablation pattern has a strong dependence on the structure of the biological tissue, which often incorporates blood vessels or fibrotic capsules and therefore results in asymmetrical ablations, which can potentially fail to reach the entire tumor. The deployment of a sensor network can potentially be used to trigger a closeloop feedback to the ablation, changing the parameters of the RF source and of the ablation device as a function of sensors readout. Such concept, namely, smart ablation, has been only partially initiated with TC sensors [21] but can be disrupted with OFS technologies.

The paper is arranged as follows: Section 2 describes the RFA setup, procedure, and measurement methodology; Section 3 outlines the measurement setup and the experimental results of Bragg grating-based measurements (FBG and LCFBG); Section 4 outlines the DTS setup for distributed measurement along multiple axes; Section 5 describes the pressure measurement and its significance; Section 6 discusses the measurement setups and their potential use in a smart RFA concept; finally, Section 7 draws conclusions.

\section{RFTA Setup}

Figure 1 illustrates the setup used for RFTA. The RF source is a medical-grade generator (TAG $100 \mathrm{~W}$, Invatec), with $480 \mathrm{kHz}$ sinusoidal carrier. The emission power is kept constant throughout the experiments, within the 9-20 W range in order to adjust the duration of ablation and the power density per unit of volume at the ablation point; most experiments have been carried out using $20 \mathrm{~W}$ electrical power. The RF generator embeds an impedance meter, which records the electrical impedance of the tissue in real time and disconnects the RF power when it falls outside the range 20-300 $\Omega$. Both voltage and current provided by the RF generator are recorded with an oscilloscope (Agilent Technologies DSO3062A, $60 \mathrm{MHz}$ ), in order to monitor the RF power input to the ablator.

RFTA experiments have been performed on samples of porcine liver, the natural phantom of the human tissue. In agreement with $\mathrm{EU}$ protocols, all specimens originated from pigs slaughtered no more than 48 hours in advance of the experiment and are commercially available; samples have been refrigerated until the ablation. The impedance of the tissues chosen for experiments is $60-120 \Omega$ at ambient temperature; during the heating process, impedance slowly decreases (about 15\%) until temperature reaches a plateau around $\sim 95^{\circ} \mathrm{C}$. Then, the water constituents of the liver transition from liquid to vapor state, and, as a consequence, the tissue impedance abruptly rises to several $\mathrm{k} \Omega$, causing the disconnection of the RF power and the interruption of the RFTA. This phenomenon is particularly evident in hepatic ablations due to the high concentration of watery constituents in the tissue, which cause an almost instantaneous rise of the electrical impedance in excess of one order of magnitude.

In some experiment, the liver sample has been positioned in a chamber built with extruded polystyrene (XPS) material, in order to confine the tissue in a finite volume preventing its expansion, as in Figure 1; other experiments have been designed leaving the explanted tissue external to the chamber.

The ablation device used in most experiments is a hollow brass stem-shaped needle with $3 \mathrm{~mm}$ inner and $4 \mathrm{~mm}$ outer diameter. The needle sustains the penetration in the tissue 


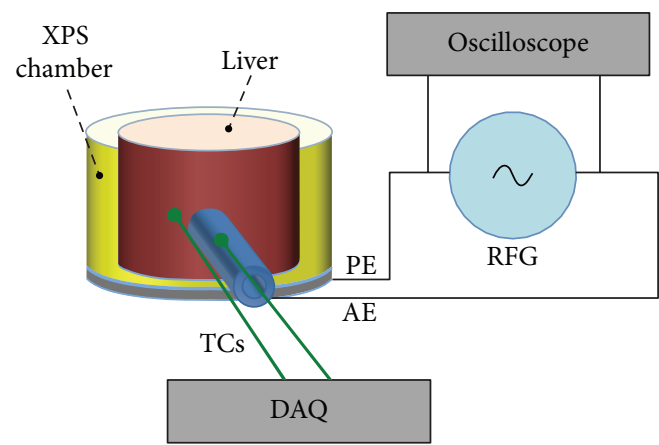

FIGURE 1: RFTA setup. A radiofrequency generator (RFG) is used to power an ablation device having $3 / 4 \mathrm{~mm}$ inner/outer diameter and with $1 \mathrm{~cm}$ active length on the tip; the active electrode (AE) is connected to the ablator, while the passive electrode $(\mathrm{PE})$ is connected to a plate below the measurement chamber, in a neutral spot. RF power is monitored in real time through an oscilloscope. The measurement chamber, made of extruded polystyrene (XPS), is designed in accordance to each experiment and internally hosts the porcine liver tissue. Two thermocouples (TCs) are used as reference, one positioned internally to the ablator, one positioned externally in the tissue, and both controlled with a data acquisition (DAQ) system in LabVIEW.

and has an electrically active electrode with $10 \mathrm{~mm}$ length on its tip, providing the electrical connection with the tissue. The needle is connected to the RF generator, while a plate positioned below the chamber is the second electrode; the difference of potential is therefore applied between the needle tip and the reference plate. In order to provide a temperature reference, a TC $\left(0.1^{\circ} \mathrm{C}\right.$ accuracy) has been positioned within the hollow ablation device, in correspondence of the center of the electrode.

\section{Fiber Bragg Grating-Based Sensors}

3.1. Fiber Bragg Gratings. FBG arrays represent the most straightforward technique for inline temperature measurement with fiber-optic technology [32-35]. By using an FBG array fabricated on a drawing tower [36,37], it is possible to reduce the distance between adjacent sensors and building a sensor array with constant spacing between each active region. This allows an unambiguous labeling of the sensors at the point of treatment.

The setup for FBG sensing, illustrated in Figure 2, makes use of an array of 5 draw-tower FBGs (FBGS DTG-1550) fabricated on ormoceramic bend-insensitive fiber. Each active length is $5 \mathrm{~mm}$, interleaved by $5 \mathrm{~mm}$ void, obtaining a sensing structure with 1 sensor/cm spatial resolution. FBGs have Bragg wavelengths around $1550 \mathrm{~nm}$, with $1.8 \mathrm{~nm}$ spacing between adjacent FBGs, and $11.66 \mathrm{pm} /{ }^{\circ} \mathrm{C}$ thermooptic coefficient [26]. Sensors are interrogated with a white-light setup, based on a broadband source (Optolink ASE, $20 \mathrm{~mW}, \mathrm{C}+$ $\mathrm{L}$ bandwidths) coupled to a spectrometer (Ibsen I-MONUSB) for detection. The use of a grating array fabricated on a drawing tower, whereas the gratings have both uniform spacing in the spectrum, with identical wavelength separation at constant temperature and along the longitudinal axis of the fiber, allows a straightforward mapping of each measurement point.

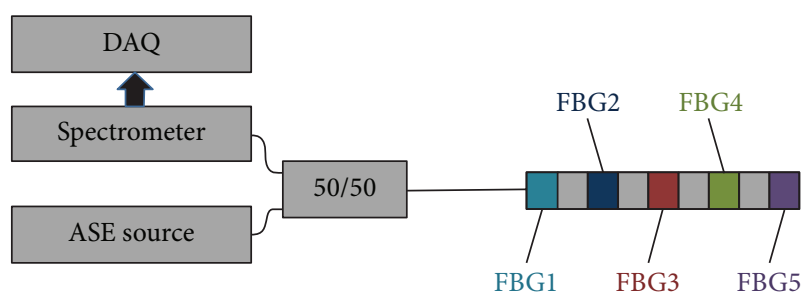

(a)

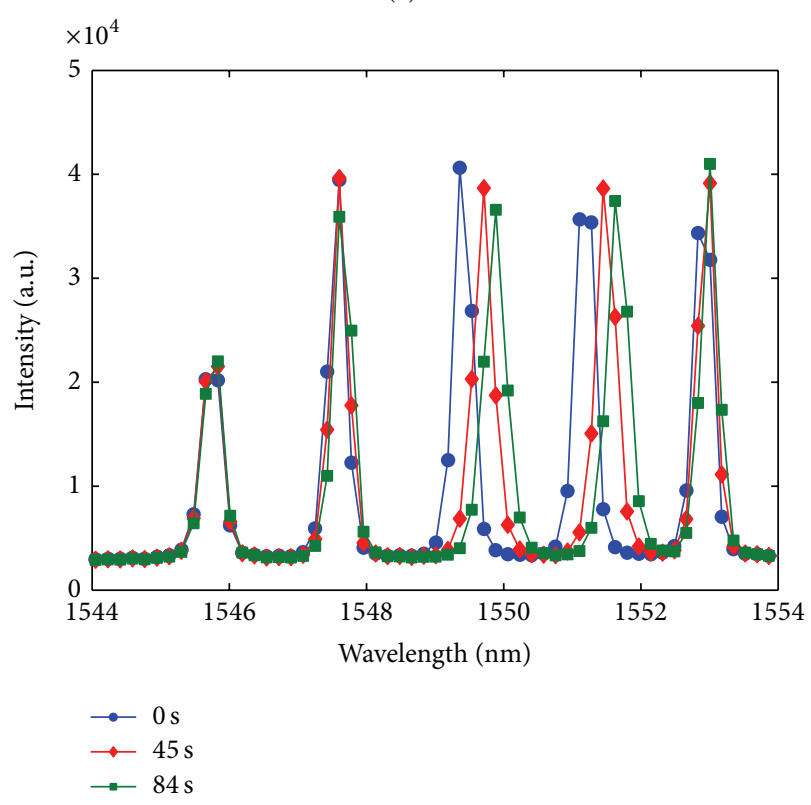

(b)

FIGURE 2: (a) Schematic of the FBG interrogation setup, showing the labeling of each FBG on the array. (b) FBG array spectrum after $0 \mathrm{~s}$, $45 \mathrm{~s}$, and $84 \mathrm{~s}$ elapsed in a thermal ablation procedure.

Accurate demodulation of the FBG array is performed in the spectrum domain. In first place, the pitch wavelength of each FBG is tracked by means of a spectral correlation technique as in [38], with $0.07^{\circ} \mathrm{C}$ typical accuracy on each of the 5 measurement points. Then, using a spline function, the spatial resolution is artificially expanded from $1 \mathrm{~cm}$ to $1 \mathrm{~mm}$ by interpolating the inner measurement points. Figure 2(b) shows the spectrum of the FBG array, as recorded by the spectrometer, through a sample RFA procedure.

Experiments with the FBG arrays have been performed with two setups, photographically shown in Figure 3 . The aim of this approach is estimating the axial temperature distribution (i.e., along the direction of the ablation device), whereas the thermal gradient is lower $[15,26]$. The first setup mimics the insertion of the FBG array on the ablation device, as in Figure 3(a). In this setup, the ablation peak is located in correspondence to FBG4 (as labeled in Figure 2(a)). The second setup is a better representation of the FBG array inserted externally through the tissue, and the ablation peak is located within FBG2 and FBG3. All experiments have been carried out using a $4 \mathrm{~mm}$ hollow ablation device and $20 \mathrm{~W}$ RF power.

Figure 4(a) shows the result of the ablation performed with the first setup. After the initialization, temperature 


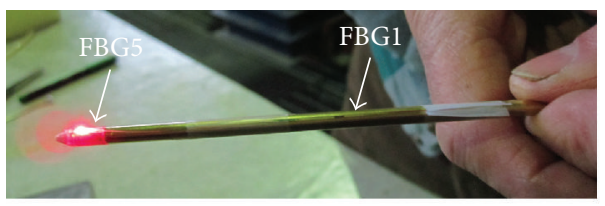

(a)

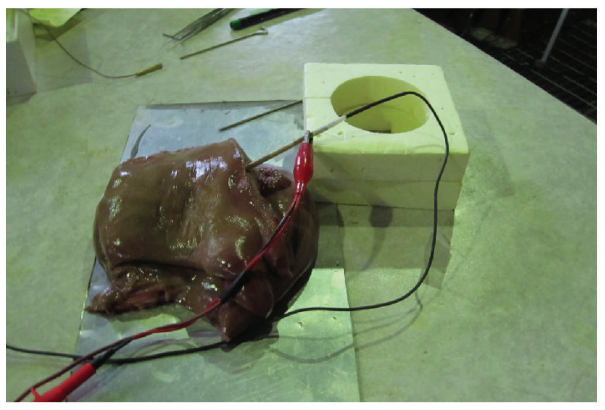

(b)

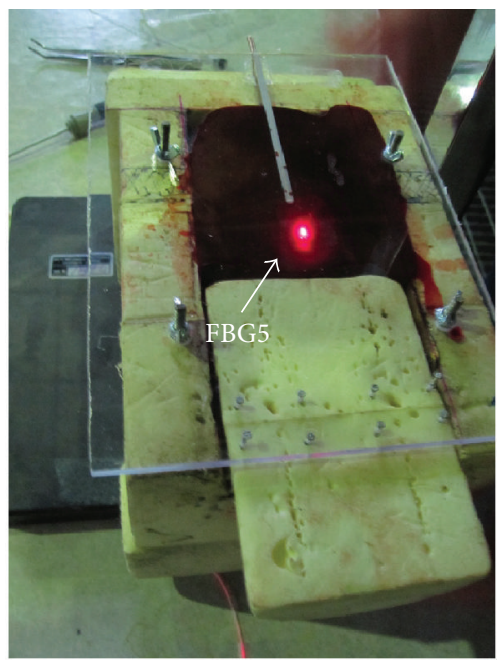

(c)

FIGURE 3: Measurement chambers used for FBG sensing in RFA, mimicking the FBG array embodied in the ablation device ((a)-(b)) and an external insertion (c). In the first setup, the FBG array (a) is attached to a $4 \mathrm{~mm}$ ablation device, stem-shaped, patching the fiber outside of the active electrode; the ablator is then inserted (b) in porcine liver lying on a metal plate serving as passive electrode. In the second setup (c), an extruded polystyrene chamber $(10 \times 8 \times 6 \mathrm{~cm})$ is set up, confining the liver tissue. A plexiglass layer encloses the chamber from the top, having the FBG array attached on its bottom side. A red laser is used to illuminate the fiber tip.

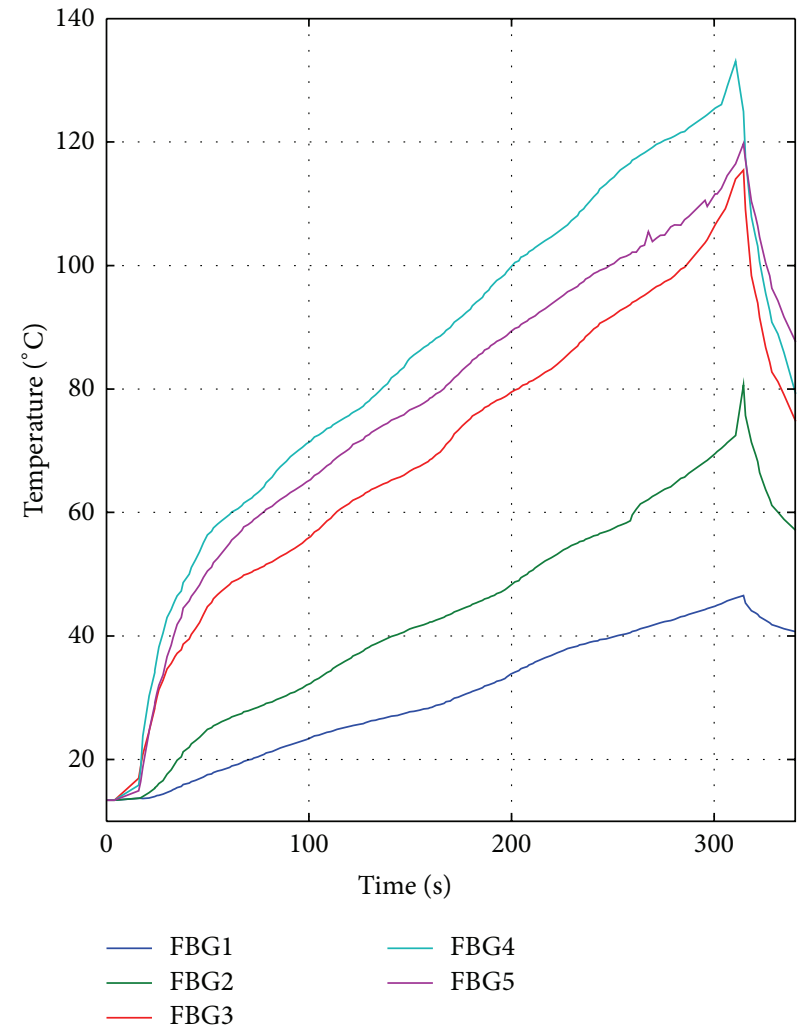

(a)

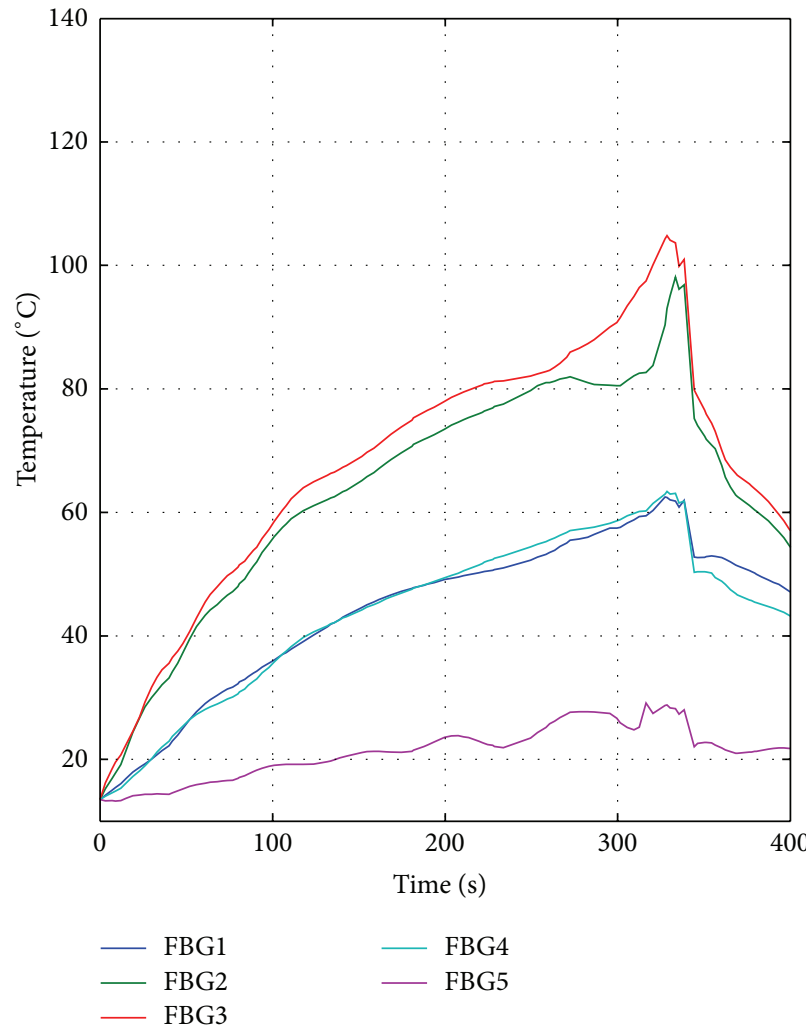

(b)

FIGURE 4: Temperature recorded by the 5 FBG sensors throughout two RFA procedures. (a) RFA performed using the setup as in Figure 3(a). (b) RFA performed using the setup as in Figure 3(c). 
$\left({ }^{\circ} \mathrm{C}\right)$
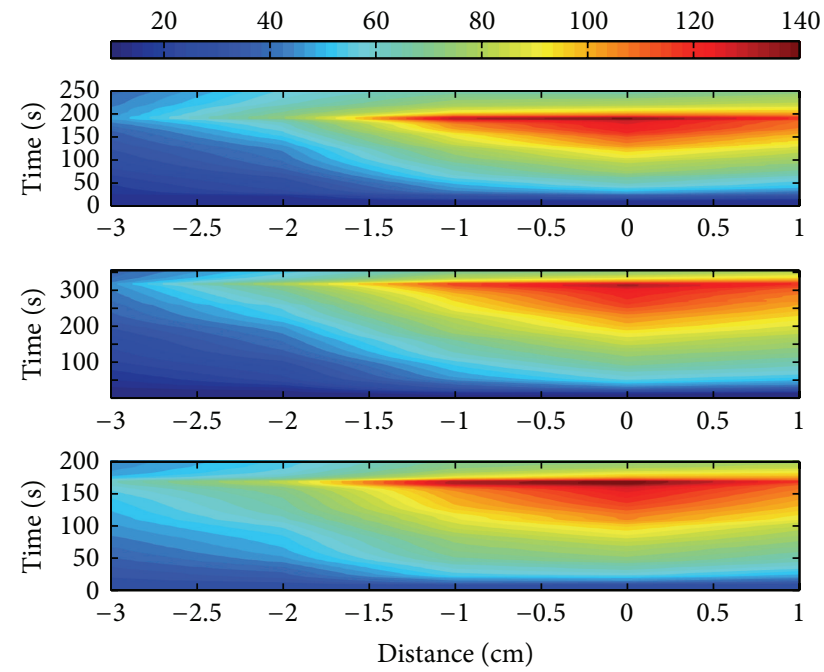

FIgURE 5: Temperature distribution (color bar, in ${ }^{\circ} \mathrm{C}$ ) along longitudinal axis for three different RFA experiments, performed with the setup as in Figures 3(a)-3(b) and with constant RF power.

increases more than linearly in the first part of the ablation, reaching the $60^{\circ} \mathrm{C}$ threshold [9] after $51 \mathrm{~s}$ at the ablation peak (FBG4) and $111 \mathrm{~s}$ for FBG3. In a second phase, temperature grows almost linearly, with an approximate gradient of $0.35^{\circ} \mathrm{C} / \mathrm{s}$ for $\mathrm{FBG} 4$ and $0.26^{\circ} \mathrm{C} / \mathrm{s}$ for FBG3 within the $80-240 \mathrm{~s}$ interval. A final temperature rise is recorded, and temperature exceeds $100^{\circ} \mathrm{C}$; at this stage, the rapid impedance increase of the tissue causes the RF generator to discontinue the power supply, and temperature starts to decrease. The temperature recorded by FBG1 and FBG2 is substantially lower [14, 15], as a reflection of the thermal gradient. Both charts show a similar ascending trend, with an extended linear range; FBG2 records a maximum temperature of $80.2^{\circ} \mathrm{C}$ and intersects the $60^{\circ} \mathrm{C}$ level after $255 \mathrm{~s}$, while $\mathrm{FBG} 1$ does not record a cytotoxic temperature.

Figure 4(b) shows the RFA experiment with the setup as in Figure 3(c). In this case, the ablation peak lies between FBG2 and FBG3. FBG3 records the highest temperature $\left(103^{\circ} \mathrm{C}\right)$; unlike the previous experiment, it exhibits a nonlinear growth in proximity of the ablation peak, which typical with the variability of RFA procedures. The chart shows that the final rise of temperature is experienced by FBG3 earlier than FBG2, with a delay of about $35 \mathrm{~s}$. FBG1 and FBG4, positioned almost symmetrically with respect to the ablation peak, record a very similar pattern, and the temperature recorded by these sensors just overcomes the $60^{\circ} \mathrm{C}$ threshold in correspondence to the ablation peaks.

Ablation data can be reported as a thermal map, after upsampling temperature on the longitudinal axis. Such format, as in Figure 5, allows also a visual comparison between different ablation experiments. Contours in particular show the temperature pattern, which is approximately quadratic in the first experiments, almost linear in the second experiment, while, in the third RFA, the linear trend appears to be altered in proximity of FBG4.
Time and temperature normalization allow a quantitative comparison between different RFA experiments. The normalization takes into account the start of the RFA procedure and the final instant of the heating phase, before the RF power is disconnected; these initial and final values of temperature are normalized between the [0-1] range. The same normalization will be used for all the experiments.

The use of FBG sensing can also enable RFA prediction features [22]. Figure 6 reports the results of the same ablation experiments as in Figure 5, and both time and temperature have been normalized. In the second ablation procedure, which substantially differs from the other experiments as it has a longer duration, it is possible to notice that the FBG positioned at the peak (FBG2) records a lower normalized temperature as compared with the other ablations. On the other side, for all the other FBGs, the normalized temperature recorded in the second experiment is higher, with a significant deviation recorded in FBG3. Data recorded in Experiments 1 and 3, which exhibit a similar behavior, are nearly overlapping, with the exception of a difference recorded in proximity of FBG4. The possibility to use the thermal data recorded by the FBG array as a metric for the effect of RF irradiance in the tissue can be exploited as a predictive asset: the thermal distribution discriminates between a heat distribution that has a deeper penetration in the tissue, as in the first and last experiment, and conditions in which the temperature field is more confined near the ablation focus, as in the second experiment, which results in a smaller ablation volume.

3.2. Linearly Chirped Fiber Bragg Gratings. While uniform FBGs are a discrete array of sensors, with a tight identification of active and passive regions, chirped FBGs [32, 34, 39-41] allow extending FBG sensing to quasidistributed detection. A chirped FBG can be modeled as a cascade of reflectors [34, 42], each having a different Bragg wavelength; when a temperature variation is experienced in each part of the grating, the corresponding local pitch wavelength shifts linearly as for uniform FBGs [17]. By using a linear-chirp pattern, thus fabricating a LCFBG $[17,39,43]$, it is possible to have a linear relationship between each grating portion and the correspondent local temperature variation. This allows using the LCFBG as a distributed sensor having an active region corresponding to the grating length, and a spatial resolution proportional to the wavelength resolution of the spectrometer.

The setup for LCFBG interrogation is illustrated in Figure 7. The optical equipment is the same used for FBG interrogation (Section 3.1). The sensor is a LCFBG (Technica S.A.) fabricated on standard single-mode fiber and with a $250 \mu \mathrm{m}$ protective coating; Figure 8 shows the grating spectrum. The grating length is $1.5 \mathrm{~cm}$, while the $3 \mathrm{~dB}$ bandwidth is $33.4 \mathrm{~nm}(1529.3-1562.7 \mathrm{~nm})$, which corresponds to a chirp coefficient of $2.22 \mathrm{~nm} / \mathrm{mm}$. The LCFBG has a maximum reflectivity of $94.5 \%$ and $2.0 \mathrm{~dB}$ ripple. When uniform temperature is applied, the LCFBG spectrum exhibits a thermooptic coefficient of $10.2 \mathrm{pm} /{ }^{\circ} \mathrm{C}$.

The decoding of the whole temperature distribution through the LCFBG involves signal processing. Skaar's reverse layer peeling $[42,44]$ is a popular method for grating synthesis but involves overwhelming computational 

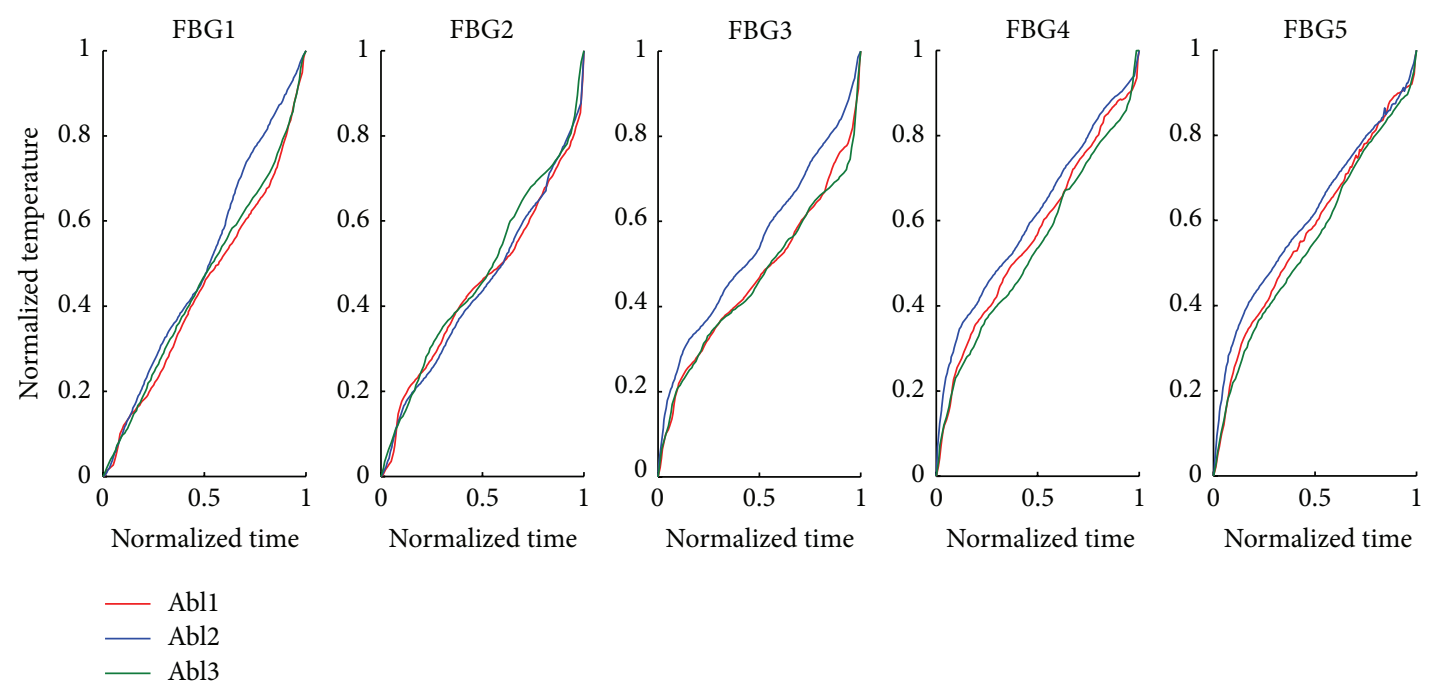

FIGURE 6: Thermal pattern of RFA, considering only temperature rise. Both time and temperature variation from the initial offset are normalized within the [0-1] range. Data are reported for each FBG sensor, for the three RFA experiments as in Figure 5.

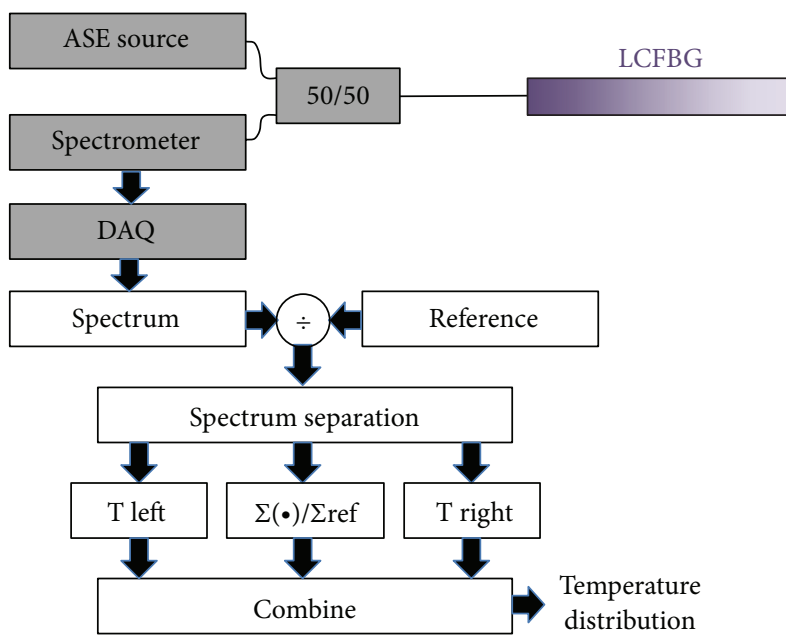

FIGURE 7: Setup for interrogation and decoding of the LCFBG. Interrogation is based on a white-light scheme, with a $1550 \mathrm{~nm}$ spectrometer for detection. The simplified decoding algorithm, as in [17], is initialized by dividing the measured LCFBG spectrum by the reference spectrum, acquired in reference condition (uniform temperature). The obtained spectral function is an almost flat curve, which depends on the whole temperature pattern, while the fluctuations at the two sides (the uppermost red and blue parts of the LCFBG spectrum) identify the temperature values at the two sides of the grating, that is, the "left" and "right" temperatures. The inner part of the spectrum is integrated and normalized for a calibration function acquired in reference condition, to estimate the thermal slope. Finally, each contribution is combined to obtain the temperature distribution through the whole LCFBG active length.

complexity and cannot be operated in real time. Other approaches involve a simplified detection, in a small number of sensing points [41] or by tracking the bandwidth [40]; alternatively, detection is performed in the time domain [39]. In order to operate temperature distributed detection

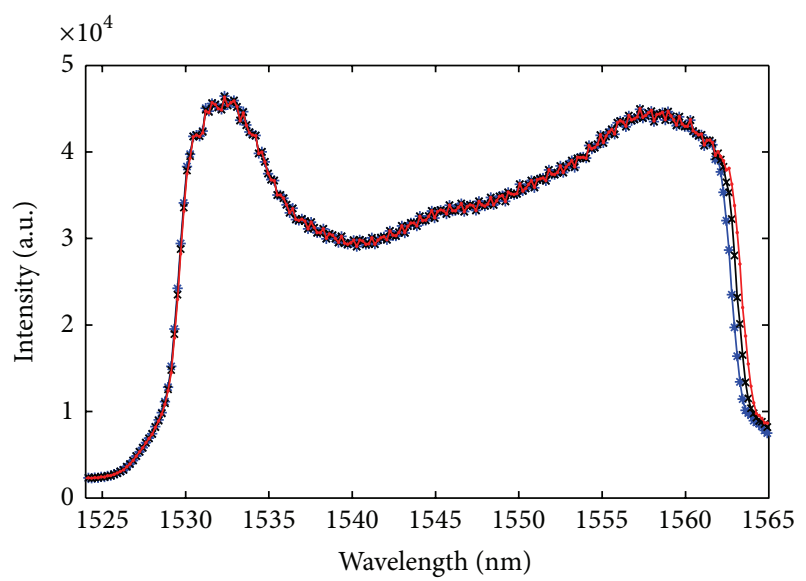

FIGURE 8: LCFBG spectrum acquired at ambient temperature (blue) and after $76 \mathrm{~s}$ (black) and $128 \mathrm{~s}$ (red) elapsed time in RFA heating.

in real time, Tosi et al. recently proposed a method based on spectral analysis, which provides a simplified detection based on simple spectral operations; this approach is sketched in Figure 7 and reported in [17] and returns the temperature with nonuniform accuracy of $0.1^{\circ} \mathrm{C}-2.5^{\circ} \mathrm{C}$ along the grating length (from the head to the tail of the sensor) and a spatial resolution of $\sim 75 \mu \mathrm{m}$ correspondent to $166 \mathrm{pm}$ resolution of the spectrometer divided by the chirp coefficient.

Since the LCFBG has a relatively short length and the capability for a narrow spatial resolving, it has been used to monitor the ablation in the direction perpendicular to the ablation axis (hence, perpendicular to the FBG array sensor as in Section 3.1), whereas the depth of penetration of heat into the tissue is lower. As for the previous characterization, two measurement setups have been used, as in Figure 9. The first setup, similar to Figures 3(a)-3(b), is based on direct insertion of the ablation device into the liver phantom; the LCFBG is 


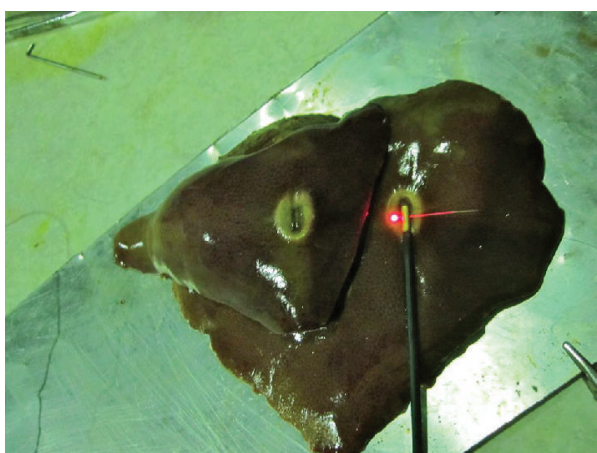

(a)

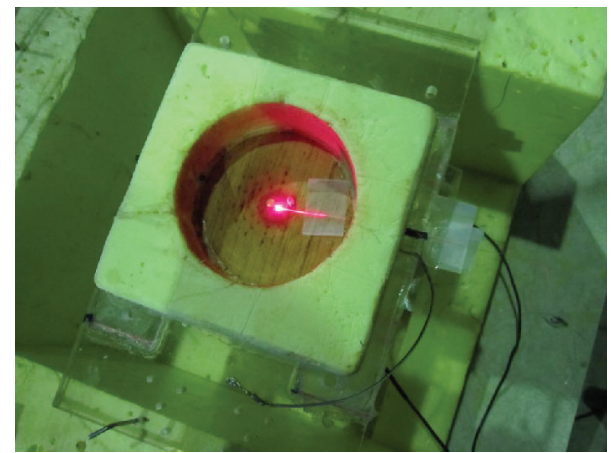

(b)

FIGURE 9: Measurement setups used for RFA with LCFBG monitoring of the temperature along the perpendicular axis to the ablation device. The LCFBG is illuminated with a red laser, highlighting the whole active part where light scatters in the outer buffer. (a) RFA has been performed on plain liver, with a $4 \mathrm{~mm}$ ablation device ( $1 \mathrm{~cm}$ active electrode). The LCFBG is positioned perpendicularly to the ablation device at the center of the electrode. The ablation device is inserted through the tissue; the picture shows the liver phantom after the RFA treatment, cut in half to show the ablation pattern. (b) Porcine liver is confined in a cylindrical XPS chamber, with diameter $6 \mathrm{~cm}$. The ablation device, same as (a), is inserted perpendicularly to the chamber plane from the bottom side. The LCFBG lies on the bottom plane, in contact with the tip of the electrode. The picture shows the chamber setup prior to the insertion of the liver phantom. RFA experiments have been performed using RF power of $20 \mathrm{~W}$.

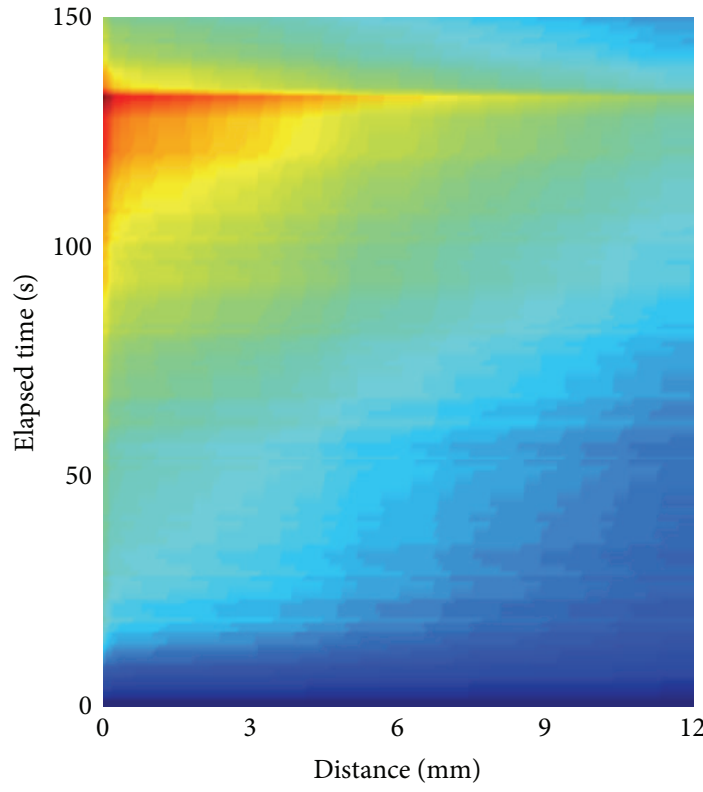

(a)

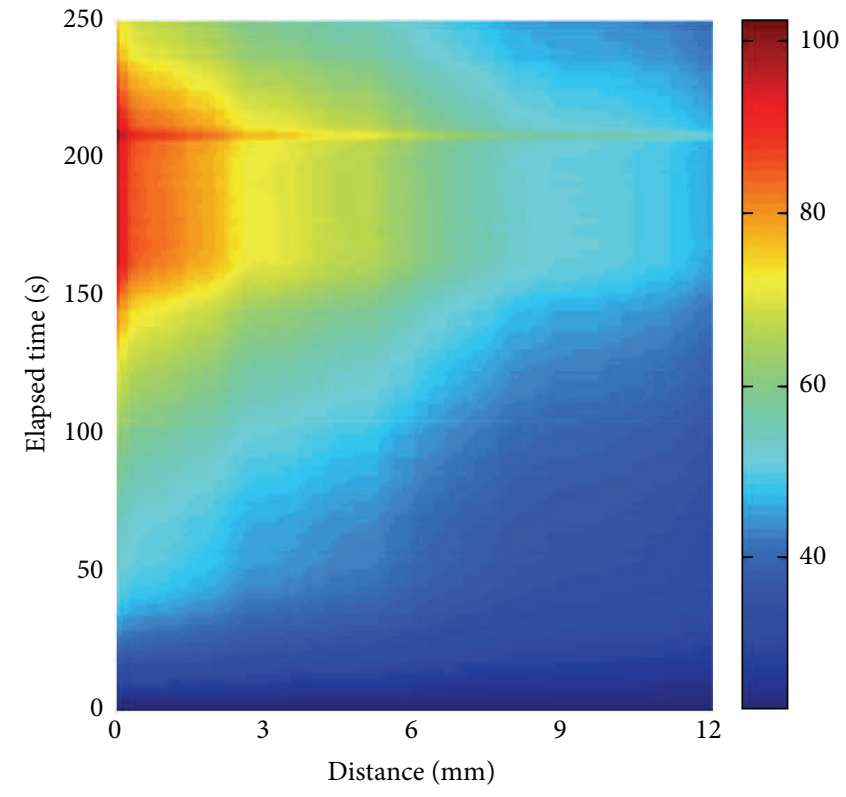

(b)

FIGURE 10: Thermal maps of RFA experiments, reporting the instantaneous temperature (color bar, in ${ }^{\circ} \mathrm{C}$ units) as a function of distance from the sensor tip and elapsed ablation time. (a) Setup as in Figure 9(a); (b) setup as in Figure 9(b).

positioned perpendicularly to the direction of ablation, in contact with the active electrode. The second setup, closer to Figure 3(c), encloses the porcine liver in a cylindrical chamber; ablation is performed on the bottom plane of the chamber, and the LCFBG sensor is fixed perpendicularly to the ablation device on the bottom plane, in contact with the electrode.

Figure 10 shows the results of two RFA experiments, carried out with the setups as in Figure 9; temperature is reported as a function of distance (measured from the
LCFBG tip, positioned in contact with the electrode) and time. The qualitative analysis of the thermal maps shows similarities with the measurements recorded in Figure 4. The first chart shows an almost linear temperature trend, similar to Figure 4(a) and Experiments 1 and 3 in Figure 5. The narrow spatial resolution of the LCFBG highlights that the approximate linear trend occurs both in space and time, with approximate gradient $2.8^{\circ} \mathrm{C} / \mathrm{mm}$. This linear trend, as confirmed by the previous measurement, confirms that the depth of penetration of heat through the tissue is higher: 

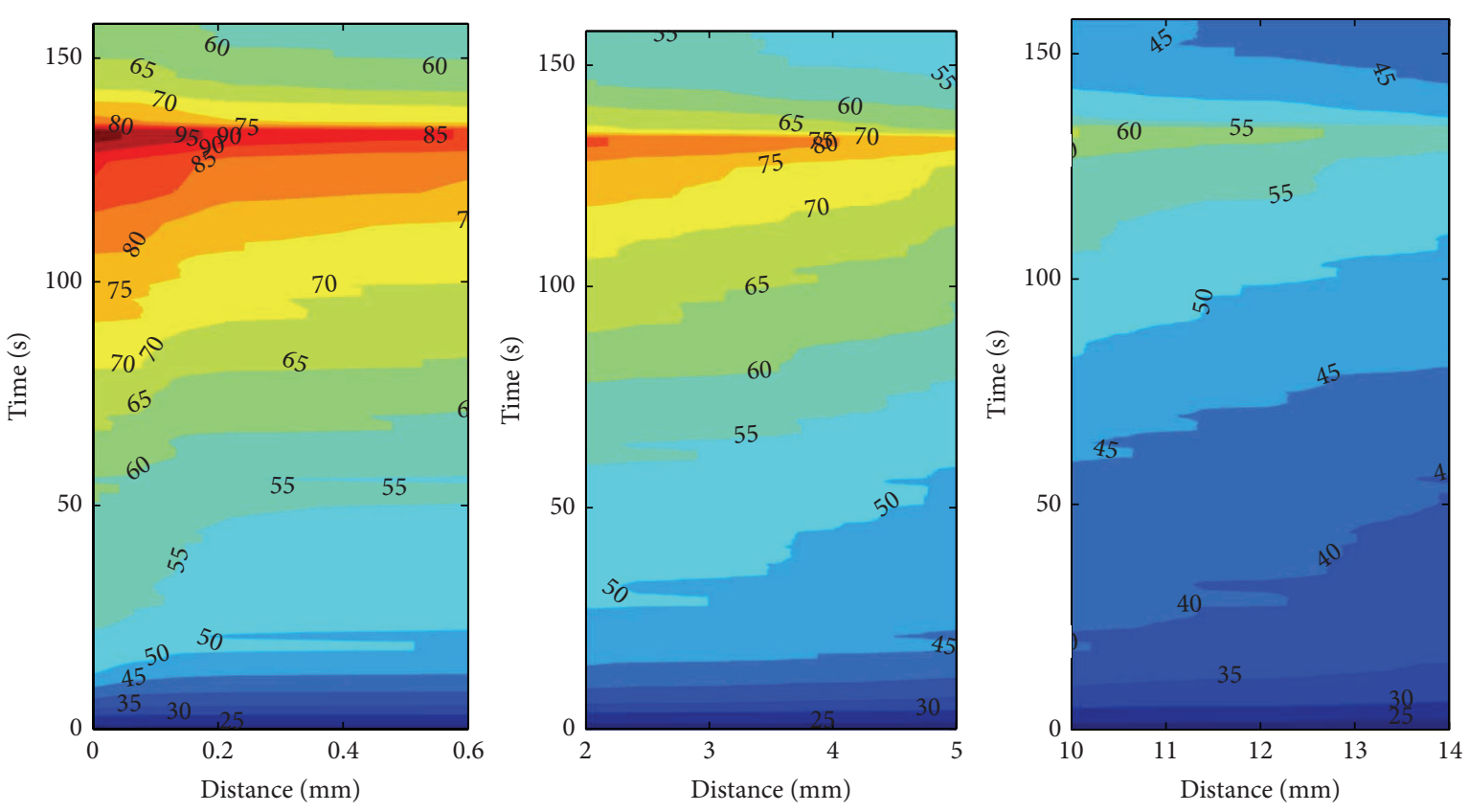

Figure 11: Contour view of Figure 10(a); the distance axis has been split between low (0-0.6 mm), mid- (2-5 mm), and long (10-14 mm) distance; numbers on each chart display temperature in ${ }^{\circ} \mathrm{C}$.

a temperature higher than $55^{\circ} \mathrm{C}$ is obtained through all the distances up to $12 \mathrm{~mm}$ before the RFA reaches the final abrupt temperature rise. On the other hand, the second chart shows a trend more similar to Figure 4(b): temperature has an almost quadratic rise over time, particularly close to the ablation peak, and the spatial distribution is nonlinear, with a perceivable distortion with respect to Figure 4(a). Visually, the thermal map appears almost flat along the distance axis, particularly within the $0.2-2.6 \mathrm{~mm}$ region. The $55^{\circ} \mathrm{C}$ threshold is achieved only for distances lower than $11.2 \mathrm{~mm}$, which results in a lower effectiveness for tumor treatments. Figure 10, in line with [15, 22, 45], highlights that the ablation pattern has a strong variability, and it depends on the measurement chamber setup.

In order to emphasize quantitative results, Figure 11 reports the contour plot as in Figure 10(a). For short distances $(0-0.6 \mathrm{~mm})$, temperature is rapidly growing reaching a maximum of $104^{\circ} \mathrm{C}$ within $0.08 \mathrm{~mm}$ from the peak point. The middistance $(2-5 \mathrm{~mm})$ range entirely overcomes the $60^{\circ} \mathrm{C}$ threshold after $88 \mathrm{~s}$ well before the end of the RFA treatment. The thermal gradients in this range are nearly linear and almost constant. The long-range (10-14 mm) plot shows that the $60^{\circ} \mathrm{C}$ value is reached only for distance lower than $10.4 \mathrm{~mm}$. This region defines the margin of ablation, and the accurate measurement of temperature can define the mortality rate of cancer cells [9].

\section{Distributed Temperature Sensing}

Distributed sensing provides an alternative approach over grating-based sensors [46-50]. DTS systems turn an entire fiber cable into a fiber sensor, without discrimination between active and passive regions. Optical time-domain reflectometry is a typical approach for achieving long-distance sensing [46-48], over multi-km range; on the other side, optical frequency-domain reflectometry (OFDR) [51-54] provides a technique for distributed sensing with a narrow spatial resolution.

The OFDR method proposed by Froggatt et al. [51-53], based on coherent demodulation of Rayleigh backscattering, allows achieving narrow spatial resolution, as low as $20 \mu \mathrm{m}$, over fiber length higher than $20 \mathrm{~m}$ [52]. The principle of operation is based on swept-wavelength interferometry (SWI) [53]. A tunable laser is used as light source, splitting the laser beam through a coupler between a measurement arm (connected to the fiber output to the DTS) and a reference arm (internal to the DTS unit); a second coupler recombines the two arms. Light is split into two orthogonal polarization states by means of a polarization controller between the two arms $[50,53]$ and a polarization beam splitter on the reference end. The SWI setup records the chaotic Rayleigh backscattering pattern, and, by means of the polarizationbased setup, it is capable of spatial discrimination over a narrow line width. When the fiber under test experiences a temperature variation, it is possible to observe a red shift of the Rayleigh signature with a coefficient $\left(10.0 \mathrm{pm} /{ }^{\circ} \mathrm{C}\right)$ similar to Bragg grating sensors; using a correlation method as in [53], it is then possible to obtain the temperature variation over the whole fiber length.

This principle of operation is implemented by the instrument (OBR 4600 DTS, provided by Luna Technologies) used for DTS sensing. The DTS achieves a minimum resolution of $20 \mu \mathrm{m}$ with a maximum fiber length of $20 \mathrm{~m}$ and up to $1 \mathrm{~Hz}$ sampling rate. Performances are, however, a result 

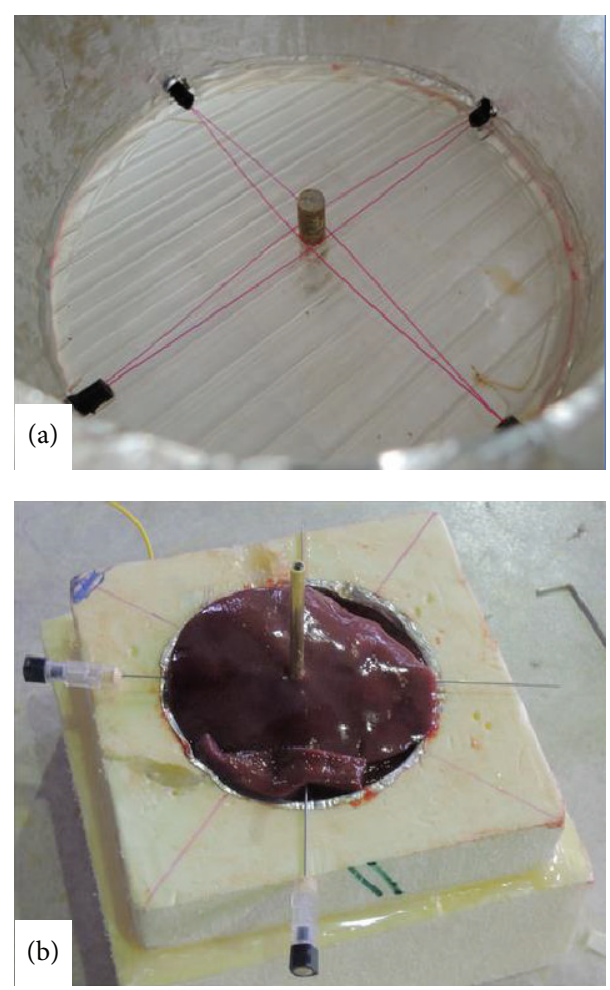

FIGURE 12: Photographs of the measurement chamber used for DTS experiments. (a) Measurement chamber prior to liver phantom positioning; fibers have been colored to show their positioning; (b) picture of the chamber after positioning of the liver phantom and ablation device.

of a tight trade-off between such parameters; in order to match the best RFA specifications and achieve real-time monitoring with $1 \mathrm{~s}$ measurement rate, the spatial accuracy has been set to $200 \mu \mathrm{m}$. With this setting, the temperature accuracy is estimated to be $0.2^{\circ} \mathrm{C}$. The spatial resolution can be narrowed in postprocessing to $20 \mu \mathrm{m}$, by correlation analysis of the whole Rayleigh patterns; this feature requires extensive computations and is not available in real time. A thermistor is used to acquire the initial temperature in the tissue.

The instrument calibrated in two steps, in first place, a fiber with $20 \mathrm{~m}$ length and $\sim 100 \%$ broadband reflectivity, is used to generate the reference Rayleigh signature, and subsequently the DTS is calibrated for the thermal coefficient after the fiber is positioned in the phantom.

The setup for DTS measurement, as shown in Figure 12, exploits the long-range sensing for monitoring RFA along several ablation axes. The ablation device ( $4 \mathrm{~mm}$ diameter, $1 \mathrm{~cm}$ electrode, as in Figures 3(a) and 9) is inserted from the bottom side of a cylindrical measurement chamber having $72 \mathrm{~mm}$ diameter. One single-mode fiber (Corning SMF28 ) is connected to the DTS and performs temperature sensing. The fiber intersects the chamber along multiple axes, lying on the ablation plane, as in Figure 12(a); this setup allows measuring the temperature along 8 radii. Liver phantom is then positioned on top of the ablation plane, as

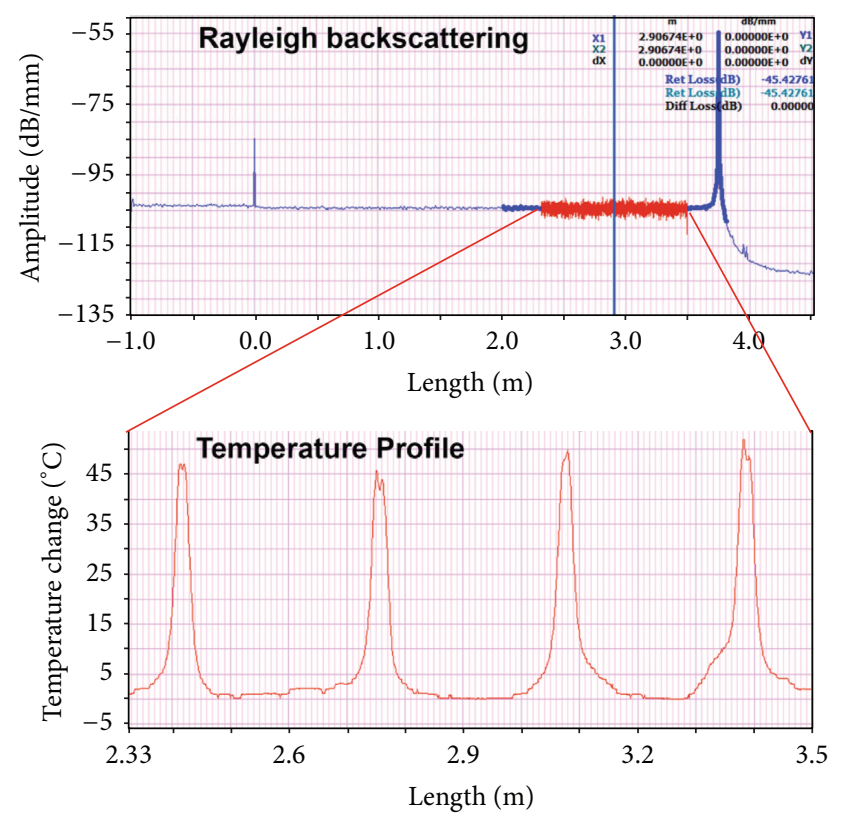

FIgURE 13: Principle of operation of DTS measurement. The chart shows data recorded during a RFA experiment. The upper chart shows the Rayleigh signature of the whole fiber span used in the measurement. The DTS connector at $0 \mathrm{~m}$ length is visible, as well as the not cleaved output connector at $3.75 \mathrm{~m}$ distance from the DTS output. The red part of the chart contains the Rayleigh backscattering acquired in the fiber portion including the parts of fiber enclosed in the measurement chamber as in Figure 12. This part of the trace is processed by the DTS to estimate the temperature variation (bottom chart); the 8 measurement radii are visible as 4 thermal peaks.

in Figure 12(b). The measurement principle of this setup is shown in Figure 13: the DTS records the temperature profile over the whole fiber length, and the only portion including the RFA monitoring is processed to estimate the temperature variation.

Figure 14 reports the thermal map observed for each radius measured from the ablation tip, for a RFA procedure with $15 \mathrm{~W}$ electrical power having $269 \mathrm{~s}$ duration. The threedimensional chart shows an evident similarity between the different directions; only during the liquid-to-vapor transition, which is the least predictable part of RFA [45], a perceivable difference between the different directions can be observed. Temperature recorded by the DTS system has a similar pattern with respect to thermal maps recorded with FBG and LCFBG sensors (Figures 5 and 10); in particular, temperature exhibits a quadratic increase during the first part of RFA, until reaching a plateau around $90-95^{\circ} \mathrm{C}$, and then rapidly grows before the $\mathrm{RF}$ generator discontinues power supply.

In order to better evaluate the shape of temperature distribution, Figure 15 reports the temperature pattern as a function of distance from the ablation device. Temperature shows an initial rapid growth, followed by a slower increase that runs through the majority of the RFA duration. During this phase, the temperature distribution follows a constant pattern, compatible with $[15,45]$. As temperature approaches 

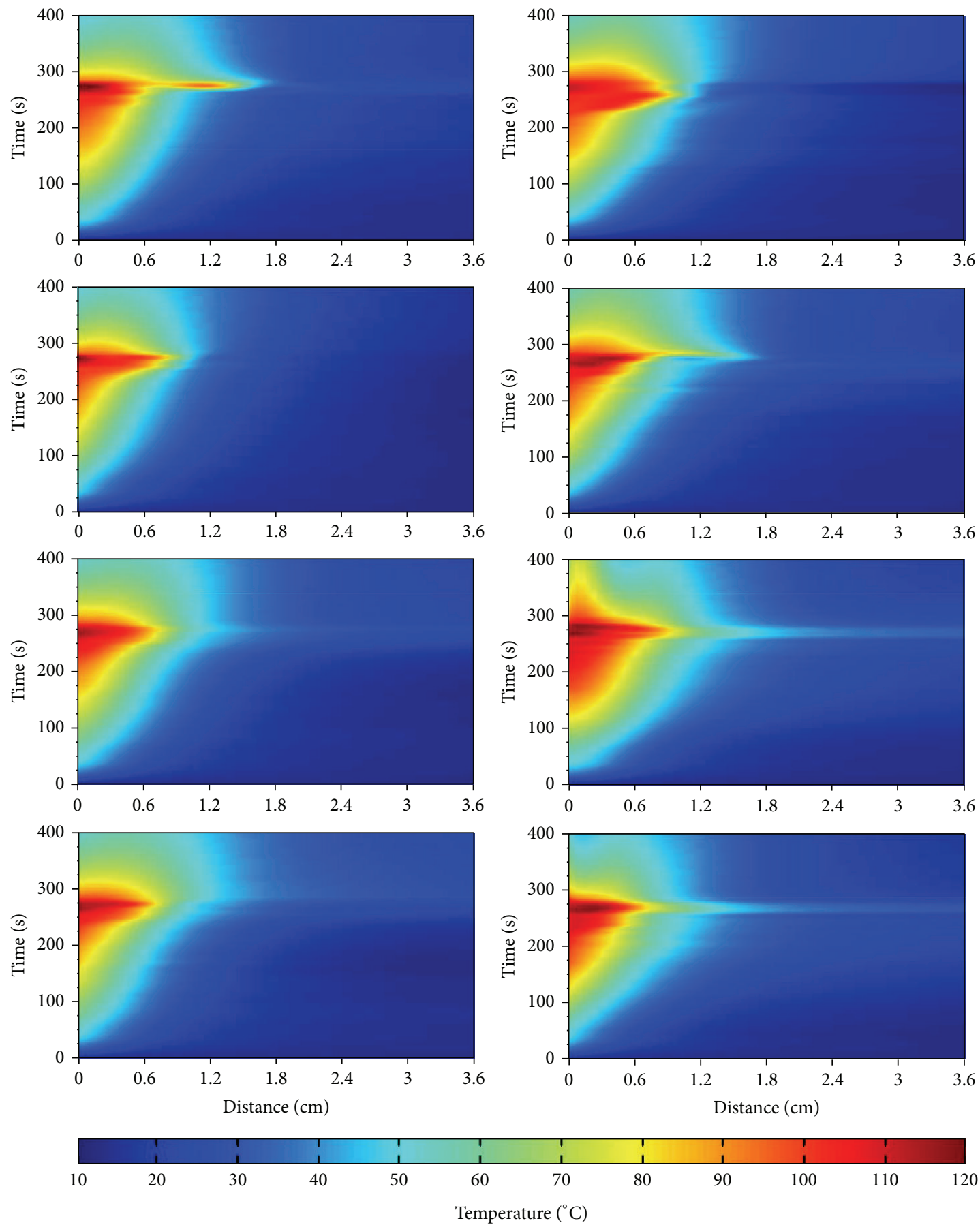

FIGURE 14: Evolution of a RFA treatment, with $15 \mathrm{~W}$ RF power, over 8 different radii. For each radius monitored with the DTS, the temperature map is reported as a function of the distance from the ablation tip and elapsed time.

the final plateau preceding the RF power suspension, it is possible to observe a change of shape in the spatial distribution, as the heat field appears to increase its depth of penetration in the tissue, particularly for the $7-9 \mathrm{~mm}$ interval.

In order to evaluate the effect of the "average" RFA, Figure 16 reports the instantaneous temperature, average over 4 ablations performed with the setup as in Figure 12 on different porcine phantom samples and with the same RF power, as a function of elapsed time; the temporal axis is normalized in order to average the different ablations. The chart shows, similar to Section 3.1, that temperature rise has a different slope as a function of distance from the ablation device tip. It is also possible to notice that the ablation peak occurs with increasing delay moving away from the ablation point.

The setup reported in Figures 12-16 exploits the capability of the OFDR system for distributed sensing; this setup is 


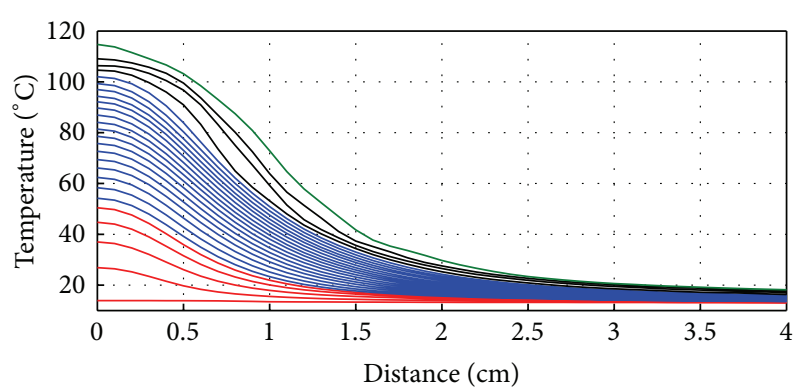

FIGURE 15: Temperature reported as a function of distance from the ablation tip, for the RFA experiment reported in Figure 14. The instantaneous temperature is reported with $10 \mathrm{~s}$ time interval, during the temperature rise phase; data are spatially averaged over the 8 radii. Red curves show the initial rapid heating with a quadratic trend, which reduces to an approximately linear rise (blue). After reaching a plateau, the thermal field increases the depth of penetration (black), until reaching the final stage (green).

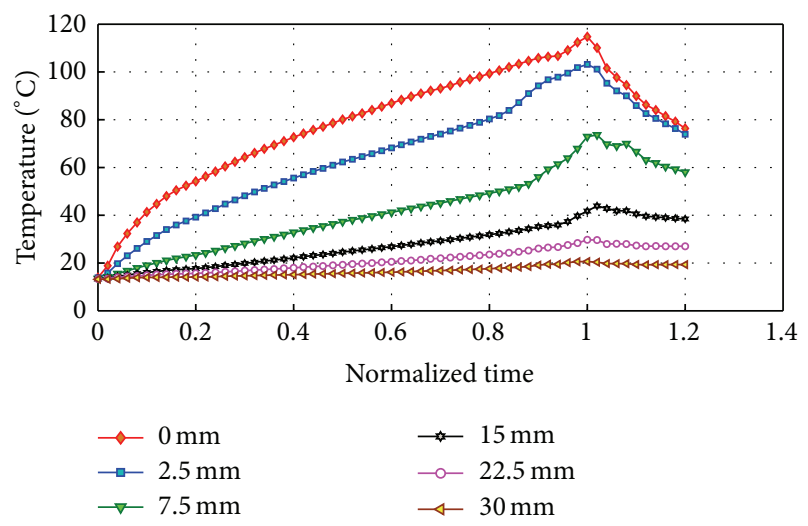

FIGURE 16: Temperature reported as a function of time (normalized to the $0-1$ interval, at $0 \mathrm{~mm}$ distance) for 6 different distances from the ablation device. The results are average between 4 different ablation procedures.

a powerful tool for multiaxes measurement. In order to validate the possibility to use the DTS in vivo, a simple measurement setup that mimics Figure 3(c) has been used, using the same ablation device as in Figures 12-16 and $15 \mathrm{~W}$ power and having the sensing fiber externally inserted on the ablation plane along the longitudinal axis, as in Figure 3(c). Temperature has also been recorded with two thermistors (B57861 Epcos, Munich, Germany; accuracy $\pm 0.2^{\circ} \mathrm{C}$ ), one positioned internally to the hollow ablation device and one positioned externally at $3 \mathrm{~cm}$ distance (Figure 17).

The result shows that the DTS provides a different response to thermal variations compared to the electrical thermistor $[15,22,27]$. This is mainly due to the positioning of the thermistor inside the ablation device and the absorption of irradiance on the active sensing part [26, 27]. Such effect is partially mitigated by the higher active volume. The DTS provides a response similar to Figure 16, and it exhibits a lower response time. Measurement shows that the ablation outcome results in a small volume being treated: while

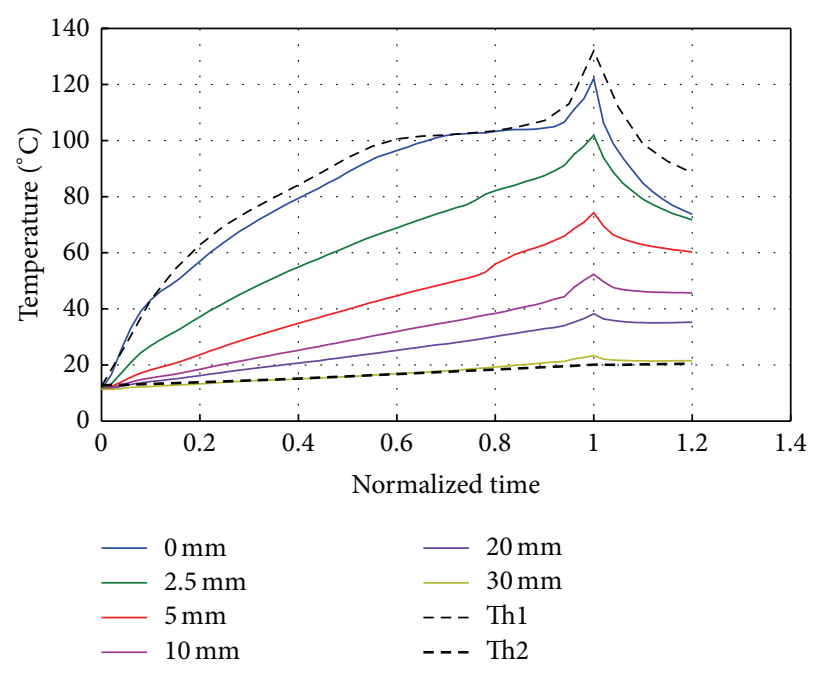

FIGURE 17: Temperature reported as a function of normalized time, for a single RFA experiment where the sensing fiber records temperature on the longitudinal axis. Data recorded with the DTS are reported for 6 different distances and compared to two thermistors.

the $5 \mathrm{~mm}$ curve clearly shows the temperature exceeding the $60^{\circ} \mathrm{C}$ threshold, the $10 \mathrm{~mm}$ curve achieves a maximum temperature of $51^{\circ} \mathrm{C}$ in correspondence to the ablation peak.

\section{Fabry-Perot Interferometry Pressure Sensing}

5.1. Significance and Sensing Challenges. The measurement of pressure in RFA is an asset for the advanced treatment of encapsulated tumors, such as hepatocellular carcinoma (HCC) [13-16, 55], which are tumors enclosed by fibrotic connective tissues, forming a cavity between the tumor tissue and its enclosing capsule. When treated via RFA, the ablation of encapsulated tumors shows a more asymmetrical pattern $[45,56]$, and the ablation procedure is less effective than in tissues having a more uniform depth of penetration. Pressure is a key metric for advancing RFA in encapsulated tumors. Tanaka et al. [56] have first introduced the intratumor pressure measurement as a parameter for RFA. However, the pressure measurement in thermal ablation, particularly RFbased, has encountered significant technological challenges mainly due to sensors characteristics.

Kotoh et al. [29] developed a setup for the intratumor pressure estimation, recording pressure values higher than $750 \mathrm{kPa}$. A microelectromechanical sensor (MEMS) has been used for pressure recording; however, the MEMS has been positioned at $3 \mathrm{~cm}$ distance from the ablation tip. This setup succeeds in providing a temperature-independent measurement, as the sensor is positioned outside of the area subjected to ablation, but it does not provide pressure readout at the point of treatment. Nakamuta et al. [57] have improved the measurement methodology using a phantom that models encapsulated HCC and demonstrated pressure measurement in RFA using a setup similar to [29] with a multitile ablation 
device [58]. References $[29,57]$ show the possibility to advance the RFA treatment, in a multistep procedure based on pressure recording; such approach is not performed in vivo, as pressure recording is not implemented at the point of treatment and MEMS sensors are not suitable for this application.

Pressure sensing in RFA monitoring is an overwhelming challenge, which requires significant advancement in sensor technologies. In particular, pressure probes need to address five specifications.

(1) Both the active and passive part of the sensor, including its cables, need to have a miniature footprint, in order not to alter the ablation pattern. Sensor/cable diameters in excess of $0.25 \mathrm{~mm}$ can significantly change the profile of heat irradiance, either when made with dielectric material (e.g., glass fibers, which can electrically insulate the surrounding tissue) or conductive material (e.g., metal packaging of sensors [28], which can bias the irradiance in the cable direction altering the symmetry of the ablation pattern).

(2) The sensor has to be biocompatible for in vivo use; typical reference standard is ISO 10993 [59].

(3) The sensor acts in close proximity to the RF ablation tip; hence, MRI compatibility needs to be guaranteed. The use of a small sensor is also advantageous as a small active volume results in a lower biasing induced by RF irradiance on the sensor tip.

(4) The sensor needs to be thermally insensitive. With most sensing technologies, including MEMS and FOS, pressure probes are cross-sensitive to temperature; in RFA, both temperature and pressure exhibit steep variations throughout the duration of the measurement; hence, thermal insensitivity needs to be guaranteed. On the other side, temperature gradients occurring at the point of RFA treatment, as documented in Sections 3 and 4, make it extremely arduous to develop a thermal compensation technique.

(5) The probe needs to have mechanical strength sufficient to sustain the penetration into the tissue and the extraction upon completion of RFA.

MEMS pressure sensors, as $[29,57]$, are typically MRI compatible and biocompatible, but they are temperature compensated only up to $\sim 50^{\circ} \mathrm{C}$ and their footprint has excessive size compared to the volume of tissue exposed to RFA. Fiber-optic sensors [25] represent a more valuable alternative for this task.

Extrinsic Fabry-Perot interferometry (EFPI) [18, 30, 60$65]$ is the most popular principle of operation for fiber-optic pressure sensing. EFPI sensors are substantially designed as a Fabry-Perot cavity on the tip of an optical fiber, sealed with a pressure-sensing diaphragm [30,60-66]. By using optical fibers, MRI compatibility is guaranteed $[30,59]$, and the size of the sensor is suitable for RFA. On the other side, the vast majority of the solutions proposed for EFPI sensors fail to address altogether the other challenges for RFA sensing.
In the first place, sensors based on epoxy, typically UV curable adhesives [63], are often not compliant to ISO 10993 standard and not resistant to high temperatures. Viable solutions include the realization of an all-glass sensor $[60,64$, 66] that uses quartz glass or an optical fiber as diaphragm, metal diaphragm as in [67], which, however, may be biased by the absorption of RF radiation on the diaphragm, or an organic material layer as diaphragm [68]. Pressure sensitivity increases by narrowing the thickness of the diaphragm [61, 63, 64]; however, sub- $\mu \mathrm{m}$ thick diaphragms as $[66,67]$ or bendable materials [68] show excessive fragility when inserted in the tissue, even with external catheterizations.

Temperature compensation has been introduced by Bremer et al. [61, 69] in EFPI sensors, using an inline FBG sensor to compensate for temperature variations from an EFPI sensor, sensitive to both pressure and temperature; this approach has been then continued in $[18,20,63,70]$ and is currently an acquaintance for EFPI sensors. The all-glass sensor proposed in [61] has a thermal detuning coefficient $1.6 \mathrm{kPa} /{ }^{\circ} \mathrm{C}$, which is the typical value for glass EFPI probes; the epoxy-based sensor proposed by Bae and $\mathrm{Yu}$ [63] has a thermal cross-sensitivity one order of magnitude higher. In this framework, even if the FBG sensor can compensate the temperature variations, the length of the FBG and its displacement from the fiber tip containing the EFPI are not negligible; indeed, the temperature recorded by the FBG is not the same value at which the EFPI is exposed to.

In 2014, Tosi et al. [18] proposed an EFPI/FBG sensor fabrication that records the smallest cross-sensitivity, achieving a thermal detuning of $0.032 \mathrm{kPa} /{ }^{\circ} \mathrm{C}$. This design is based on the fabrication of an EFPI probe with a quartz glass capillary, over a FBG sensor previously inscribed in a single-mode fiber. A novel approach, more recently introduced by Poeggel et al. [20] reverses the fabrication process, inscribing a $1 \mathrm{~mm}$ FBG sensor on an EFPI; this approach allows inscribing the grating of the exact tip of the probe, leaving no passive regions between the EFPI and the FBG. These approaches allow pressure sensing in RFA, as they comply with all the requirements for operation in thermal ablation.

5.2. Fabrication and Characterization. Figure 18(a) shows the fabrication of the EFPI/FBG probe $[18,61,63,69,70]$. In the first place, a FBG is inscribed in a standard singlemode fiber (SMF-28, 10/125 $\mu \mathrm{m}$ ), using UV laser and a phase mask [32, 71]. The grating has $4 \mathrm{~mm}$ length, with $1540.5 \mathrm{~nm}$ wavelength. After cleaving the SMF fiber in proximity of the FBG, the fiber is spliced to a glass hollow capillary, with $130 / 200 \mu \mathrm{m}$ inner/outer diameter. During this operation, the reflectivity of the FBG reduces from $95 \%$ to $4 \%$. The capillary is then hand polished $(\lambda / 10)$ and spliced to a multimode standard fiber $(62.5 / 200 \mu \mathrm{m})$ which serves as diaphragm; this procedure seals an air-gap Fabry-Perot cavity on the tip of the transmission fiber, with length $20-60 \mu \mathrm{m}$. In order to functionalize the structure for pressure sensing, the thickness of the diaphragm has to be reduced [61]: this task is accomplished by first hand polishing the diaphragm and then controlled etching in hydrofluoric acid as in [19]. All the splicing operations are performed with a manual splicer (Siecor M91). 


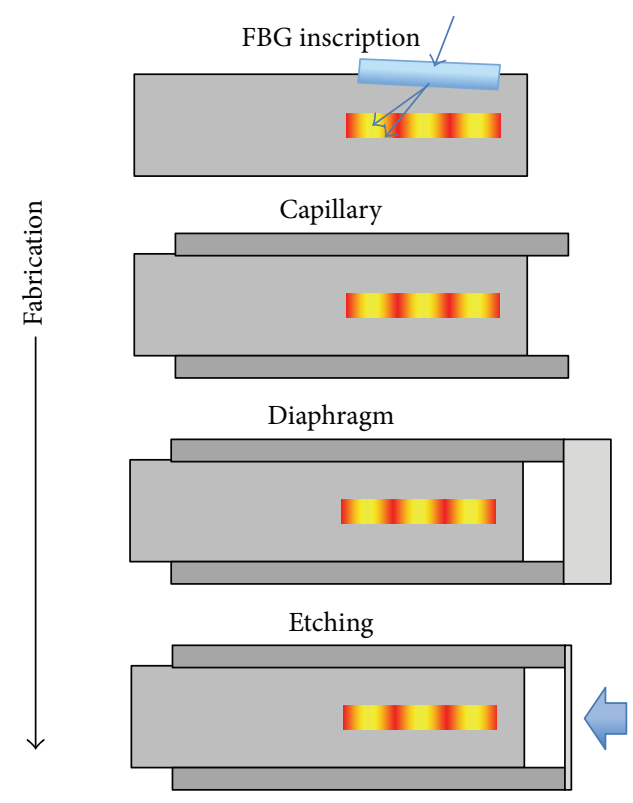

(a)

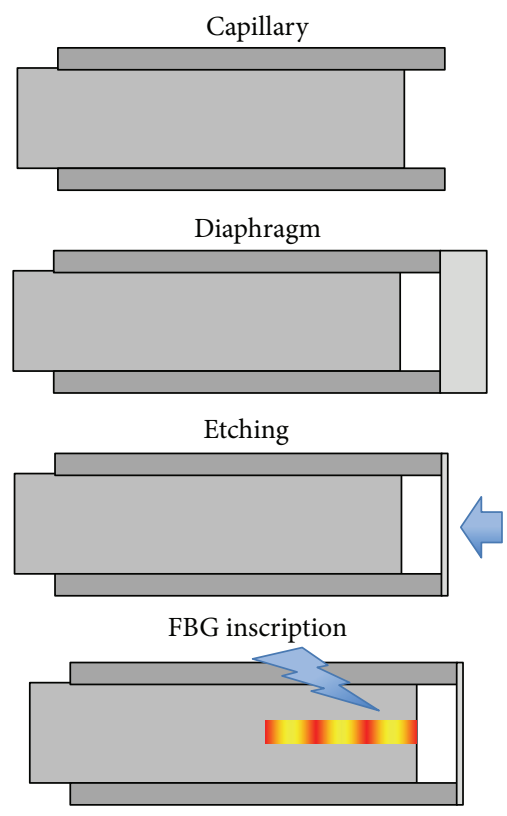

(b)

FIGURE 18: Schematic of the fabrication technique for the EFPI/FBG probe. (a) Fabrication of an EFPI probe over a preinscribed FBG sensor. In the first step, the FBG is fabricated with phase-mask technique on a standard single-mode fiber [18]; then, the fiber is spliced to a hollow glass capillary, and the resulting structure is spliced to a multimode fiber serving as diaphragm. Finally, etching in hydrofluoric (HF) acid [19] reduced the diaphragm thickness for pressure sensitivity. (b) Fabrication of an EFPI probe with subsequent inscription of the FBG: in this case, the sensor is built as in (a), steps 2-4, and the FBG is inscribed on the sensor tip as in [20].

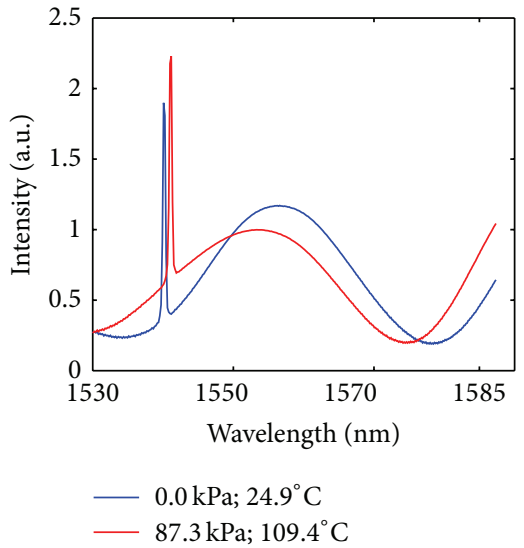

(a)

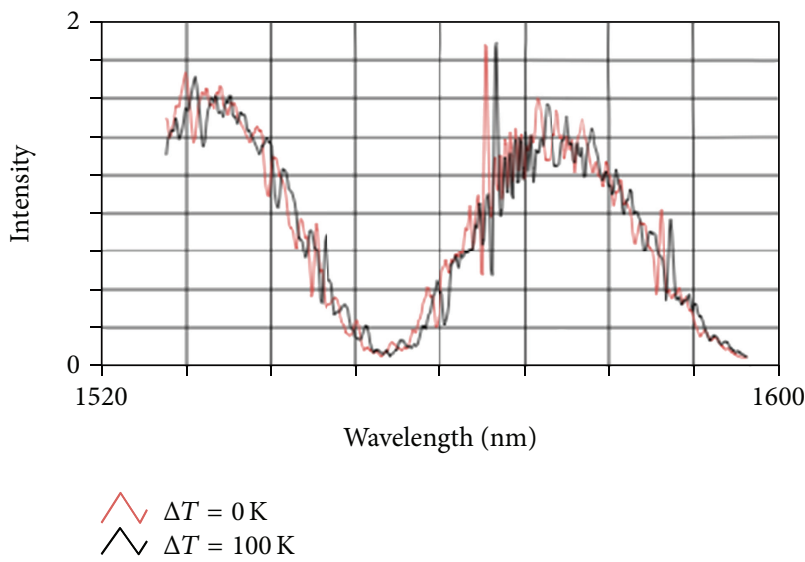

(b)

FIGURE 19: EFPI/FBG probe spectra. (a) Sensor fabricated with the classical method, as in Figure 18(a); (b) postinscription of FBG with the point-by-point method, as in Figure 18(b).

An alternative fabrication technique has been recently demonstrated by Poeggel et al. [20]. The EFPI sensor is designed as in Figure 18(a), obtaining a pressure probe. Then, the FBG is fabricated using a femtosecond laser and a pointby-point inscription [72]. While the previous fabrication method cannot allow a perfect positioning of the FBG on the fiber tip, as the splicing procedure performed near the air-gap tends to damage the FBG, this novel approach allows inscribing the FBG exactly on the tip of the probe. Hence, this second approach minimizes the distance between the probe tip and the FBG tail.

Figure 19 shows the spectra of the EFPI/FBG probes fabricated with both methods. The classical inscription method provides a clean spectrum, with a neat separation of the EFPI broad spectrum from the FBG narrow spectrum. The EFPI has a low finesse $(0.083)$, given by a low $2 \%$ reflectivity. The postinscription of the FBG has the advantage of fabricating a shorter FBG on the fiber tip, more suitable for RFA; but the 


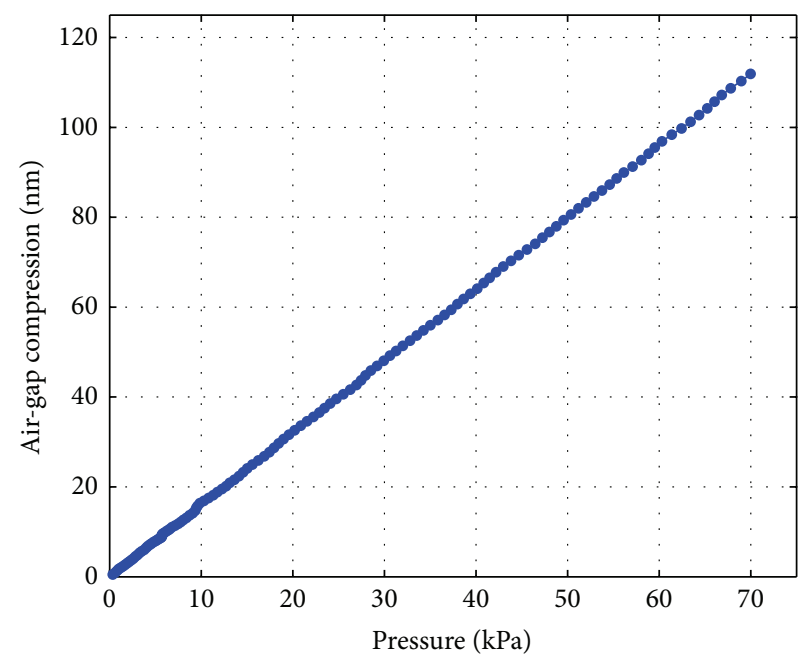

(a)

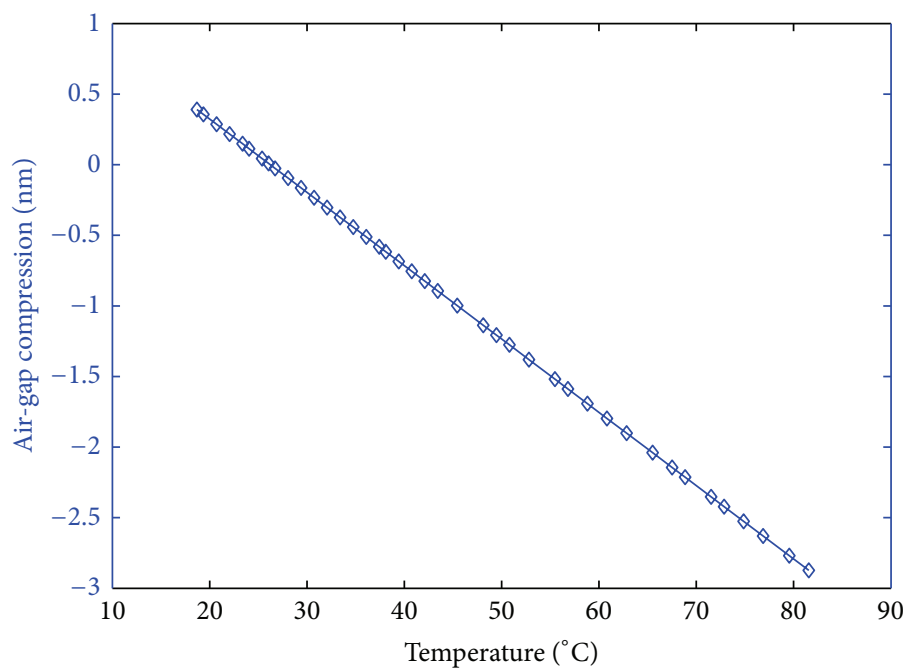

(b)

FIGURE 20: Calibration of the EFPI/FBG probe used in RFA experiments. (a) Air-gap compression as a function of the applied pressure, recorded by the EFPI; (b) air-gap compression as a function of applied temperature.

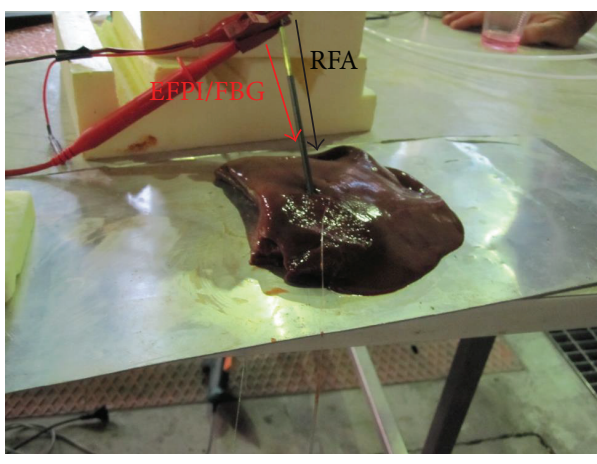

(a)

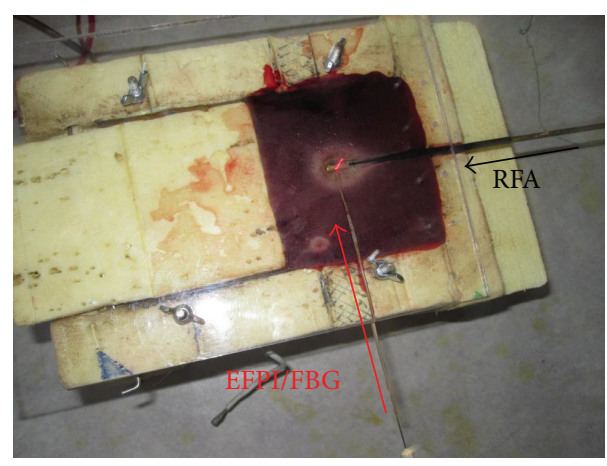

(b)

FIGURE 21: Experimental setup for pressure/temperature measurement in RFA procedure. (a) EFPI/FBG probe inserted through a hollow ablation device in plain liver phantom. (b) Fiber-optic probe inserted perpendicularly to ablation direction through an external catheter; liver tissue is confined in an XPS chamber.

resulting spectrum has a more wrinkled pattern [72]. It is still possible to discriminate the FBG from the EFPI, but pressure measurement achieves better accuracy when based on a clean spectrum.

A probe fabricated with the classical method has been used in RFA experiments; Figure 20 shows the compression of the air-gap as a function of the applied pressure $(0-70 \mathrm{kPa}$ range). The EFPI sensor has a sensitivity of $1.60 \mathrm{~nm} / \mathrm{kPa}$, compatible with $[61,63]$, and linearity coefficient 0.9997 . The choice of the glass capillary, as outlined in [18], reduces the thermal sensitivity of the EFPI to $51.9 \mathrm{pm} /{ }^{\circ} \mathrm{C}$, over 17 $82^{\circ} \mathrm{C}$ range. This value is over 30 times smaller than that in [61] $\left(1.7 \mathrm{~nm} /{ }^{\circ} \mathrm{C}\right)$ and 300 times smaller than that in [63] $\left(15.8 \mathrm{~nm} /{ }^{\circ} \mathrm{C}\right)$. The FBG has a thermooptic coefficient of $10.7 \mathrm{pm} /{ }^{\circ} \mathrm{C}$ and negligible pressure sensitivity [63]. This sensor achieves dual pressure/temperature measurement with accuracy of $40 \mathrm{~Pa}$ and $0.1^{\circ} \mathrm{C}$, respectively.
5.3. Experimental Results. Two experimental setups (pictures in Figure 21) have been designed for RFA experiment, following the approach used for FBG, LCFBG, and DTS sensing. In the first setup, RFA is performed with a hollow needle, $3 \mathrm{~mm}$ diameter and $1 \mathrm{~cm}$ active length, which penetrates the tissue with $2 \mathrm{~cm}$ approximate depth. The EFPI/FBG probe is inserted through the RFA device, and the fiber tip is located at approximately $1 \mathrm{~mm}$ distance from the tip of the active electrode. This setup mimics Figures 3(a)-3(b) and 9(a). The second setup, which is closer to Figures 3(c), 9(b), and 12, encloses the porcine liver tissue in an XPS chamber covered with a plexiglass layer, as in Figure 3(c). The EFPI/FBG probe is inserted from the side, orthogonally to the RFA needle. The picture in Figure 21 shows the pattern of ablation after the procedure is completed. Two experiments have been performed, having the fiber tip positioned at $5 \mathrm{~mm}$ and $8 \mathrm{~mm}$ from the tip of the electrode. In order to better visualize the 


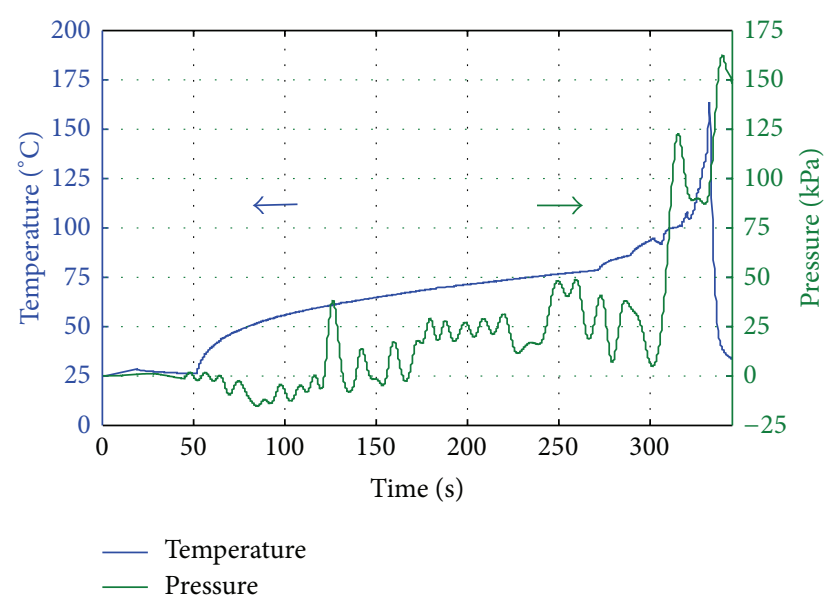

Figure 22: Dual pressure (right) and temperature (left) traces acquired by the EFPI/FBG probe through a RFA procedure with setup as in Figure 21(a).

pressure pattern, these RFA experiments have been carried out with an inferior RF power $(5 \mathrm{~W})$ to extend the duration of ablation treatments.

The result of a RFA procedure carried out with the first experimental setup is shown in Figure 22, whereas both pressure and temperature traces are displayed. After the initial setup, temperature rises quadratically (as in previous experiments), while pressure appears to be floating, initially reaching negative values $(-13 \mathrm{kPa})$ and then achieving a peak at $39 \mathrm{kPa}$. As temperature rises to $\sim 80^{\circ} \mathrm{C}$, pressure continues its oscillation reaching a maximum value close to $50 \mathrm{kPa}$. At this stage, temperature starts a rapid growth reaching a maximum value of $164^{\circ} \mathrm{C}$; the pressure trace, after an initial decrease to $8 \mathrm{kPa}$, rapidly increases first to $125 \mathrm{kPa}$ and then to $162 \mathrm{kPa}, 3 \mathrm{~s}$ after the temperature peak is recorded.

This chart is significantly different from the previous measurement by Kotoh et al. [29,57]. Quantitatively, the value of $750 \mathrm{kPa}$ recorded at $3 \mathrm{~cm}$ distance from the ablation tip has not been recorded, and maximum pressure recorded is $162 \mathrm{kPa}$ (1.62 bar). The trace also has a different profile: while the fluctuations can be partially explained by the change of refractive index of the medium surrounding the EFPI probe, pressure exhibits two almost instantaneous rise, the second one being recorded with a delay with respect to the temperature peak.

Figure 23 reports, for the experiment in Figure 21, the pressure values as a function of temperature (PT chart). In this plot, it is possible to distinguish how the two quantities are correlated. In the initial phase (1), temperature rises from ambient to $101^{\circ} \mathrm{C}$, while pressure fluctuates from the initial zero to negative and positive values, $-13 \mathrm{kPa}$ to $50 \mathrm{kPa}$. Then (2), the chart experiences an almost vertical transition: pressure rapidly grows to $92 \mathrm{kPa}$ within few seconds, in which temperature rises at a slower pace. In the region (3), both pressure and temperature exhibit a rapid change, in correspondence to the liquid-to-vapor occurring in the liver tissue: temperature quickly rises to its maximum value, while pressure first increases to $125 \mathrm{kPa}$ and then decreases

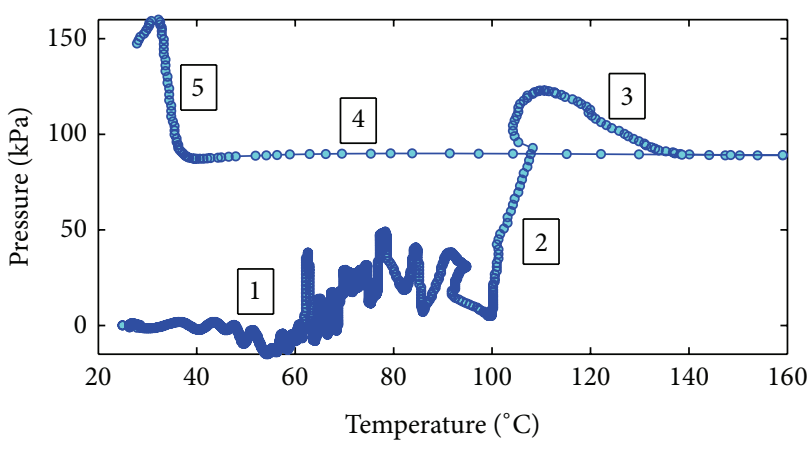

FIgURE 23: Pressure as a function of temperature, for the RFA experiment reported in Figure 21.

to $88 \mathrm{kPa}$. The last two movements on the PT chart occur at a very rapid pace: temperature drops with an instantaneous rate, corresponding to a horizontal movement (4), followed by a vertical shift (5) in correspondence to the pressure peak, occurring when the liver temperature is already $<40^{\circ} \mathrm{C}$.

The results of three RFA experiments are reported in Figure 24 reports, for the experiment with setups as in Figure 21. In first place, it is possible to notice that the pressure values are correlated with the distance from the fiber tip, as for temperature variations; maximum pressure values of $162 \mathrm{kPa}, 33 \mathrm{kPa}$, and $6 \mathrm{kPa}$ have been recorded at $1 \mathrm{~mm}, 5 \mathrm{~mm}$, and $8 \mathrm{~mm}$ distance from the fiber tip to the electrode tip $\left(31^{\circ} \mathrm{C}, 46^{\circ} \mathrm{C}\right.$, and $164^{\circ} \mathrm{C}$ maximum temperature, resp.). In both the first and second experiment, it is also possible to notice that pressure exhibits a similar trend: after an initial stabilization, with an average rise, pressure increases in correspondence to the phase transition of the liver tissue, with a slight delay with respect to temperature rise. After temperature reaches its peak, pressure continues rising and the maximum value is reached with some delay with respect to the temperature maximum. The pressure peak has been recorded for normalized time equal to 1.05 (first experiment) and 1.07 (second experiment). At $8 \mathrm{~mm}$ distance, the pressure and temperature variations are significantly smaller.

The PT chart for the RFA experiments carried out with the setup as in Figure 21(b) and with the EFPI/FBG probe at a further distance from the ablation tip shows a different pattern. Figure 25 shows the PT chart for the second RFA experiment in Figure 24. In the first place, it is possible to notice that the pattern is more linear, and the amplitude of pressure fluctuations is lower; this can be partially explained by the lower variations of refractive index (RI) surrounding the probe but also to the better enclosing of the liver that prevents pressure from rising and decaying. Compared to Figure 23, most of the trace appears to be confined in region (1), whereas both temperature and pressure rise at a steady rate. When temperature reaches the $40-44^{\circ} \mathrm{C}$ interval, pressure appears to rise linearly reaching $23 \mathrm{kPa}$ value. As temperature starts to decrease, we observe a pressure rise corresponding to a vertical transition on the PT chart; this is analogous to region (5) in Figure 25, that is, a rapid pressure rise, when temperature is reducing. Qualitatively, the chart 


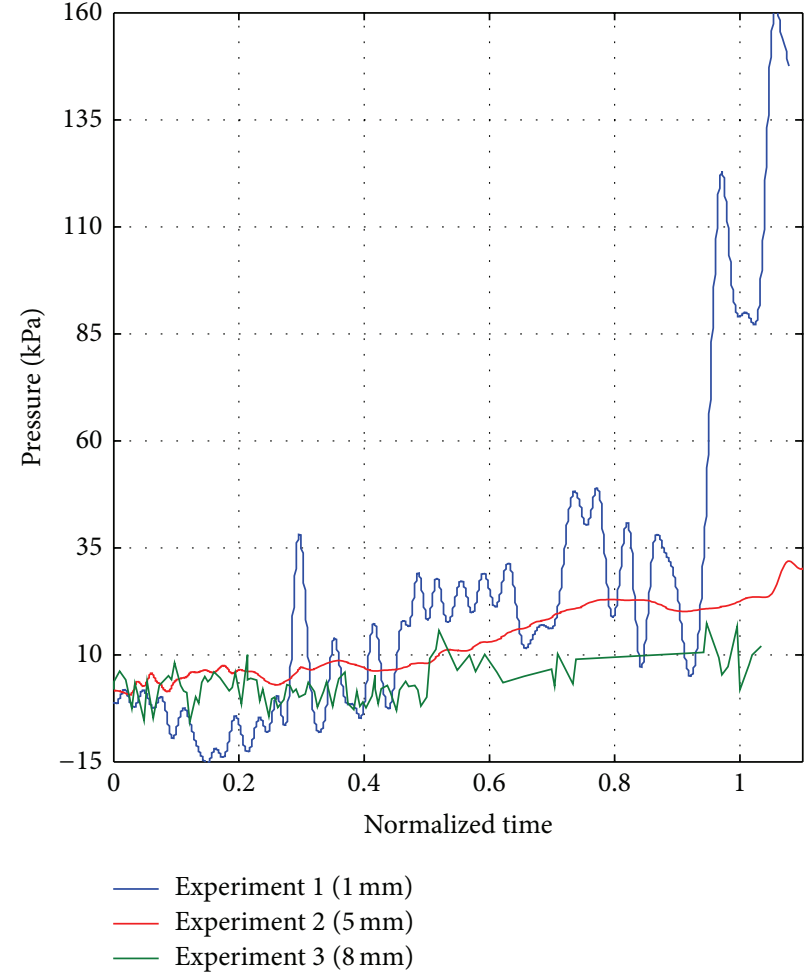

(a)

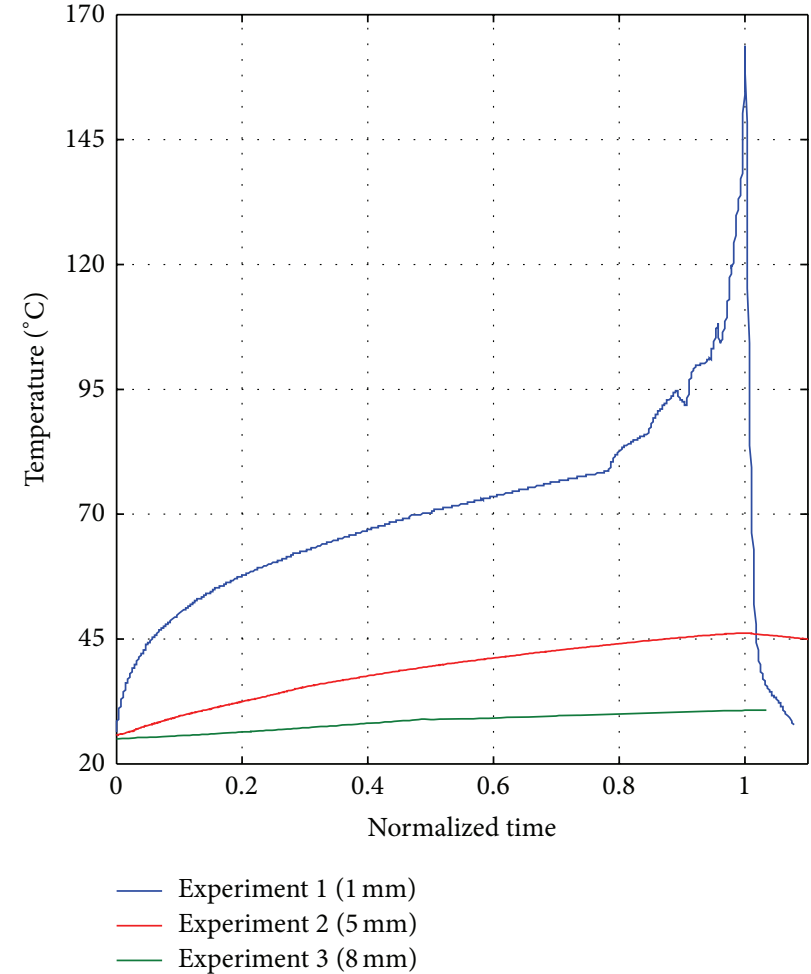

(b)

Figure 24: Pressure (a) and temperature (b) charts reported for three RFA experiments, as a function of normalized time. Experiment 1 is based on the setup of Figure 21(a), and the fiber tip is located at $1 \mathrm{~mm}$ distance from the electrode tip. Experiments 2-3 have been carried out with the setup in Figure 21(b), and the fiber tip-electrode distance is $5 \mathrm{~mm}$ and $8 \mathrm{~mm}$, respectively.

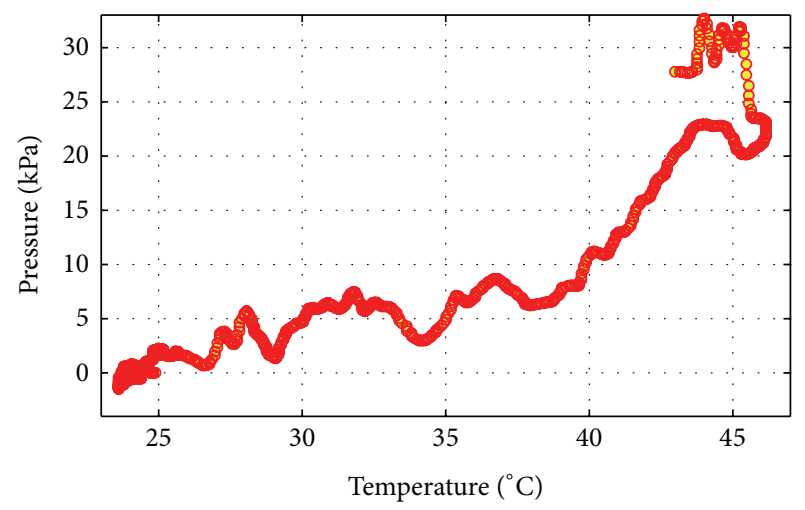

FIGURE 25: PT chart for an experiment carried out in setup as in Figure 21(b).

appears to have fewer transitions and is missing regions (1), (2), and (3).

\section{Discussion}

6.1. Technology Review and Developments. The optical fiber sensor technologies used in this work provide an allencompassing view of the possible strategies for monitoring of biophysical parameters in RFA procedures. Each approach provides different performance levels and can be dedicated to a specific task in RFA monitoring. Table 1 reviews the performance of all sensors.

FBG sensors provide the easiest way for the monitoring of a small number of sensing points, using inline sensing. FBGs have typical length of 1-4 mm, which is not negligible considering the thermal gradients generated in RFA [32]. Using standard FBGs [35], the fabrication of a sensor array is limited by splicing or other fabrication techniques to $\sim 2 \mathrm{~cm}$ spacing between each FBG. Dense FBG sensors arrays have been proposed as in [27], achieving up to 3 sensors $/ \mathrm{cm}$; this fabrication cost for such structures is however excessive for thermal ablation. The fabrication of FBGs on a drawing tower (DTGs) is a flexible alternative, which can have potential in RFA $[26,37]$. Most DTG fabrication facilities are limited to $5 \mathrm{~mm}$ minimum length and $10 \mathrm{~mm}$ minimum sensor spacing, for the fabrication of low-cost sensors; unlike traditional FBGs, the fabrication cost of a DTG is scalable with the number of sensors, as the fabrication of FBG arrays has a high fixed cost and a marginal variable cost depending on the number of sensors. The main asset of grating sensors relies on the consolidated techniques for interrogation of FBG arrays, which is nowadays implemented in a broad number of commercial devices.

At the present state of the art, the poor resolution of FBG/DTG sensors is a key limitation in thermal ablation. However, this scenario is likely to change within the next 
TABLE 1: Comparison of fiber-optic technologies used for RFA monitoring.

\begin{tabular}{lcccccc}
\hline Technology & Ref. & Active length & Spatial resolution & Accuracy & Pros & Cons \\
\hline FBG & {$[27,32-34]$} & $1-4 \mathrm{~mm}$ & $1-20 \mathrm{~mm}$ & $0.1^{\circ} \mathrm{C}$ & Inline sensor & Resolution \\
DTG & {$[26,37]$} & $5 \mathrm{~mm}$ & $10 \mathrm{~mm}$ & $0.1^{\circ} \mathrm{C}$ & Fabrication & Resolution \\
LCFBG & {$[17,39-42]$} & $1.5 \mathrm{~cm}$ & $75 \mu \mathrm{m}$ & $0.2-2.5^{\circ} \mathrm{C}$ & Resolution & Detection \\
DTS/OFDR & {$[51-53]$} & $20 \mathrm{~m}$ & $20-200 \mu \mathrm{m}$ & $0.2^{\circ} \mathrm{C}$ & Resolution & Backreflection \\
EFPI & {$[30,61,75]$} & $25 \mu \mathrm{m}$ & - & $6 \mathrm{~Pa}$ & Accuracy & Integration \\
EFPI/FBG & {$[18,20]$} & $25 \mu \mathrm{m} / 4 \mathrm{~mm}$ & - & $40 \mathrm{~Pa} / 0.1^{\circ} \mathrm{C}$ & Dual sensor & RI change \\
\hline
\end{tabular}

few years, with the advent of point-by-point FBG inscription [72] that can potentially fabricate grating structures with a narrow resolution and with potential improvements to the DTG fabrication approach $[36,37]$. Currently, DTGs are also applied for all-grating fibers [73, 74], which extend FBG sensing to an entire fiber span with distributed integration but are still limited in resolution by the spatial resolution of FBGs. Most DTG fabrications are already established for fibers with $80 \mu \mathrm{m}$ diameter, and $40 \mu \mathrm{m}$ fibers are already commercially available and soon utilizable in FBG sensors, reducing the active volume of each sensing point.

The LCFBG approach, on the other side, shows a better potential for distributed sensing [17]. It makes use of the same optical hardware as FBG sensors, using the same interrogators, but achieves a sub- $0.1 \mathrm{~mm}$ theoretical resolution. LCFBG sensors are more expensive than DTG arrays, but they use similar fabrication techniques and can benefit as well from potential improvements of point-by-point inscription; in addition, chirped gratings are commonly employed in the fiber-optic communication industry as broadband reflectors or gain-flattening filters, and therefore it is likely that, once the technology is established, their manufacturing cost can significantly reduce. The FBG used in experiment has $1.5 \mathrm{~cm}$ length, but it is possible to fabricate LCFBGs having length up to $5 \mathrm{~cm}$ and $\sim 80 \mathrm{~nm}$ bandwidth, still achieving $\sim 0.1 \mathrm{~mm}$ spatial resolution.

The main drawback of LCFBGs is that, currently, there is no reliable technique for their detection in the spectrum domain. Approaches like $[42,44]$ require an overwhelming complexity and are not affordable in real-time operation. Simplified approaches substantially turn the LCFBG into a few-point sensor [41], which does not offer significant advantages over FBGs. In particular, the main problem of all the proposed techniques, including the simplified approach proposed in this work, is that the entire spectrum needs to be detected as a whole, as the temperature estimated in each measurement point depends on the values recording in all the adjacent points.

This statement is well demonstrated in Figure 26, which compares different temperature readings performed with LCFBG, DTS, and DTG-FBG array. Although the comparison is only for qualitative purpose, as it includes different RFA experiments on several porcine phantoms and measurements are recorded along different axes, it shows that the LCFBG has a different qualitative behavior with respect to the other technologies, which however show similar readings. The simplified approach proposed in [17] for distributed temperature estimation needs to be improved in order to

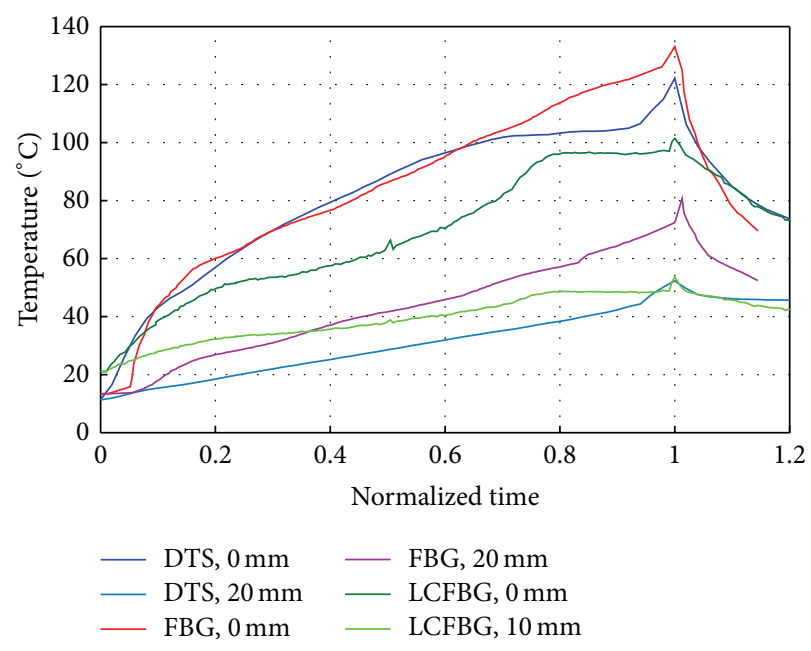

Figure 26: Comparison of different RFA experiment, recording temperature with DTS, FBG, and LCFBG technologies at different distances from the ablation tip.

make the LCFBG a reliable RFA-prone sensor. However, once such technique would be established, the sensing approach based on chirped gratings may have significant advantages over DTS: its performances (measurement rate, accuracy, and resolution) are not based on a trade-off but can be individually selected and improved by changing the optical hardware. In addition, the LCFBG sensor is not significantly affected by poor cleaving at the fiber end, which is a weakness of DTS.

The DTS, on the other side, gains the benefits of allowing distributed sensing over long fiber spans. Currently, OFDR technologies allow sensing over up to 20-70 m length and are often used for characterizing optical connectors [52]. The approach proposed by Gifford et al. [53] allows temperature sensing with $20 \mu \mathrm{m}$ (offline)- $200 \mu \mathrm{m}$ (online) spatial resolution. The main attractiveness of the DTS is that, although the instrument is expensive, having a cost that usually overcomes the RF ablation equipment, the sensor is instead a standard optical fiber, having the lowest cost among any fiberoptic technologies. Although performances depend on the tight trade-off between the different parameters, the settings used in experiments are sufficient for general-purpose RFA monitoring.

DTS is an excellent instrument for ex vivo monitoring and can be used for the realization of complex setups as Figure 12 which can really advance the state of the art of RFA modeling, 
such as the European FP7 project IMPPACT [22]. The main drawback of the DTS system is that the backreflection occurring at the fiber end substantially overlaps the trace at the fiber tip (as is clearly visible in Figure 13), which is the most significant sensing information as this part of the sensor is usually located in correspondence to the ablation tip. Although a proper fiber cleave can solve this problem, when the fiber penetrates the tissue its surface gets microdamages, neutralizing the initial cleaving quality. A possible solution is the introduction of the fiber in a microcatheter, which sustains the penetration while preserving the cleaving quality.

The three technologies presented in this work require a different calibration procedure. FBGs are linear sensors that require the characterization of the thermal coefficient, which is usually carried out prior to their use and depends almost entirely on the glass compound [33]. It is possible to obtain then the absolute temperature measurement when the sensor is initialized, either using an external sensor (ex vivo) or using the temperature reference value $36.8 \pm 0.4^{\circ} \mathrm{C}$ (in vivo). The calibration of a LCFBG requires the determination of the thermal coefficient and the temperature offset, as the FBG, but it also needs the characterization of the LCFBG spectrum in uniform heating conditions, that is, when the whole LCFBG is exposed to the same local temperature [17, Figure 7]. This characterization can be performed in a temperature chamber but may substantially differ from each LCFBG sensor as it can depend on spectral ripples, chirp coefficient, and other fabrication parameters. The DTS requires the acquisition of the reference chaotic Rayleigh signature, which is then used to estimate the wavelength shift corresponding to temperature variations in each measurement point [53]. This task is performed by calibrating the whole instrument in reference conditions, whereas the whole temperature distribution is known (e.g., constant temperature through all the fiber). However, the calibration of the instrument prior to fiber insertion is arduous, since fiber bends and eventual backreflections often occurring with the insertion of the fiber at the point of treatment may substantially detune the measurement. A simple, yet effective, calibration technique is to impose the temperature distribution in a specific portion of the fiber link, which is assumed not to have optical losses; this can be a viable solution for the application of the DTS in vivo, assuming that the temperature of the fiber is constant at the reference value $36.8^{\circ} \mathrm{C}$ and neglecting the other parts of the fiber span.

As outlined in Section 5.1, the state of the art of pressure sensor does not offer valuable alternatives to the sensor presented in this work; to the best of our knowledge, no other solution can offer thermal insensitiveness, biocompatibility, miniature size, and tensile strength, which are all essential requirements for RFA. The pressure measurements reported have more significance than [29], as they are performed at the point of treatment rather than at distance. The future work will be addressed to improving the fabrication of sensors to high-volume manufacturing rates, providing a better insulation of the fiber tip from the surrounding refractive index to avoid pressure fluctuations and advancing the clinical knowledge of advanced treatment of encapsulated tumors supported by fiber-optic probes. EFPI/FBG integrated probes

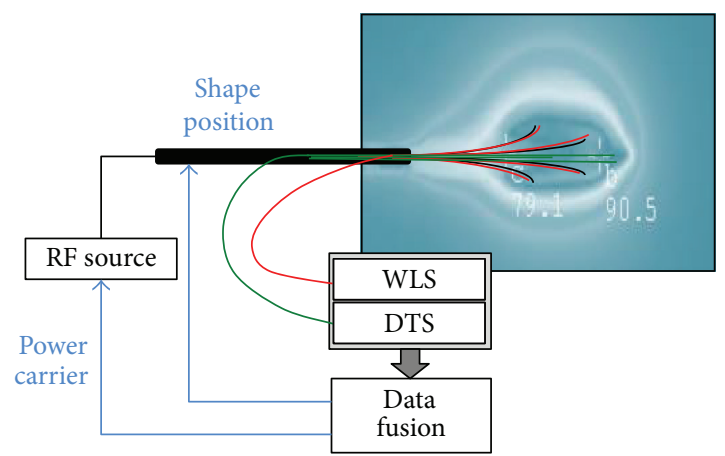

FIGURE 27: Proposal of a smart ablation concept.

offer the advantage of integrating a temperature sensor and give access to the PT chart (Figures 23 and 25). EFPI/FBG probes make use of the same interrogation setup of FBG and LCFBG sensors, and, with the technique proposed in [75], the pressure accuracy is as low as $6 \mathrm{~Pa}$.

All measurements carried out in experimental setups are based on single-channel detection; however, interrogation of 4-16 channels with optical switching can extend the sensing capabilities. Using the most recent fiber-optic switches with $20 \mathrm{~ms}$ switching time and building custom-made software for interrogation using off-the-shelf hardware, it is possible to obtain $1 \mathrm{~Hz}$ measurement rate with up to 16 channels. Such feature is not available for DTS systems, in which the measurement duration is tied to the accuracy/resolution performance levels.

6.2. Smart Ablation. The ultimate goal of the application of fiber-optic sensors in RFA is to gain the capability of performing ablation procedures based on sensors readout, rather than imaging, and dynamically change the parameters of ablation in a closed-loop way. Such technique, hereby called "smart ablation," is currently limited by the lack of sensing technologies; this work aims at filling the gap, highlighting sensing technologies that are compatible with the application in RFA. A possible implementation of the smart ablation concept is sketched in Figure 27.

Sensors can be (1) installed on each tip of a multitile device $[21,57,58],(2)$ inserted through a hollow ablation device in an electrically passive region, or (3) externally inserted with a catheter, which can eventually be removed after insertion in vivo. The latter option has been experimentally tested, with positive results [18], showing that the fiber can always be extracted from the tissue even after the catheter has been removed. A combination of LCFBG or FBG array sensors with EFPI sensors can be used. All these sensor typologies perform wavelength-selective operations and, therefore, can be all interrogated with a white-light system (WLS) operating in the near infrared, as in Figures 2 and 7, which is based on a broadband source, a coupler, and a spectrometer for spectral detection. A single WLS system can be interrogated up to $4-8$ channels, each including a combination of sensors. DTS can provide an alternative technique for temperature or strain detection, operating 
with high density over a single fiber. Combining WLS-based sensors and DTS, it is possible to record up to several hundreds of sensing data at the point of treatment, applying the lab-on-a-fiber concept with high density.

Using the sensors data, it is then possible to design a data fusion system that collects the biophysical data and uses them to adjust the parameters of RFA. The data fusion system can also incorporate imaging $[23,24]$ and modeling [22]. Haemmerich and Schutt [10] demonstrated that the ablation pattern has a dependence on the RF frequency, which suggests that smart ablation can be effective for advanced treatment of tumors. Future research will also focus on modifying the ablation device, enabling the possibility to change its position and shape dynamically through RFA, following the robotic microsurgery concepts [76, 77]. The smart ablation concept may also be extended to microwave ablation [78, 79] and cryoablation [80] of tumors.

\section{Conclusions}

The present work presents and reviews the use of optical fiber sensors in cancer thermotherapy. The specific medical scenario discussed in the present work involves the thermal ablation of liver tumors with a radiofrequency source. RFA procedure makes use of a percutaneous ablation device and hence can accept only miniature sensors such as optical fiber sensors as monitoring tool; on the other side, RFA is vulnerable, for the treatment of mid-to-large tumors, to the change of electrical impedance of the target tissue when temperatures around $\sim 100^{\circ} \mathrm{C}$ are reached in proximity of the ablation device.

The presented sensor monitors biophysical parameters: temperature distribution and pressure. Temperature distribution, sampled with the narrowest possible spatial resolution, is a key indicator of the mortality rate of cells in every portion of the tumor. Three technologies are presented: FBG arrays achieve a $1 \mathrm{~cm}$ resolution but allow straightforward measurement and interpretation; a LCFBG sensor on the other hand achieves $75 \mu \mathrm{m}$ resolution, but it is highly dependent on the interpretation of the optical spectrum, which is suboptimal with the algorithms presented to date; high-density DTS based on OFDR allows achieving resolution of $20-200 \mu \mathrm{m}$, with a tight performance trade-off (accuracy, resolution, and speed), but its performances ex vivo are hardly replicable in vivo unless special catheterizations are applied. Pressure is another key biophysical parameter, mainly for the optimal treatment of encapsulated tumors such as HCC. The authors have developed an EFPI pressure sensor, integrated with an FBG temperature sensor, which records, to the best of our knowledge, the lowest cross-sensitivity parameter. This achievement enables pressure sensing in RFA at the point of treatment, and preliminary experiments show a pressure rise in RFA procedures which depends on the distance from the ablation tip. This result is the first step towards adjusting RFA in encapsulated tumors, such as HCCs, to pressure readout.

In conclusion, optical fiber sensors can substantially advance the treatment of tumors via thermal ablation, providing real-time distributed or quasidistributed biophysical sensing at the point of treatment. The collection of such big data can be exploited to design a smart ablation system, whereas sensor readouts are used to dynamically adjust the pattern of ablation in real-time.

\section{Conflict of Interests}

The authors declare that there is no conflict of interests regarding the publication of this paper.

\section{Acknowledgments}

The authors acknowledge the contribution of Sven Poeggel, Gabriel Leen, and Elfed Lewis (University of Limerick, Optical Fibre Sensors Research Centre); Mario Gallati and Giovanni Braschi (Universita' di Pavia, Dipartimento di Ingegneria Civile ed Architettura); Giorgio Busca (Politecnico di Milano, Dipartimento di Meccanica); and Sandro Rossi (Policlinico San Matteo). The authors acknowledge Ian Shannan and Maurizio Chiani (Luna Technologies) for providing a DTS unit and Hugo Mertens, Johan Vlekken, and Eric Lindner (FBGS International) for supplying ormoceramic fibers. Research has been funded by Marie Curie actions (MC-IEF299985), Science Foundation Ireland (10/RFP/ECE2898), Enterprise Ireland (CF-2014-0107-Y), and Fondazione per la Cura Mini-Invasiva dei Tumori.

\section{References}

[1] B. J. Wood, J. R. Ramkaransingh, T. Fojo, M. M. Walther, and S. K. Libutti, "Percutaneous tumor ablation with radiofrequency," Cancer, vol. 94, no. 2, pp. 443-451, 2002.

[2] S. N. Goldberg, G. S. Gazelle, and P. R. Mueller, "Thermal ablation therapy for focal malignancy: a unified approach to underlying principles, techniques, and diagnostic imaging guidance," American Journal of Roentgenology, vol. 174, no. 2, pp. 323-331, 2000.

[3] L. Solbiati, T. Livraghi, S. N. Goldberg et al., "Percutaneous radio-frequency ablation of hepatic metastases from colorectal cancer: long-term results in 117 patients," Radiology, vol. 221, no. 1, pp. 159-166, 2001.

[4] A. Orlando, G. Leandro, M. Olivo, A. Andriulli, and M. Cottone, "Radiofrequency thermal ablation vs. percutaneous ethanol injection for small hepatocellular carcinoma in cirrhosis: metaanalysis of randomized controlled trials," The American Journal of Gastroenterology, vol. 104, no. 2, pp. 514-524, 2009.

[5] S. N. Goldberg, G. S. Gazelle, C. C. Compton, P. R. Mueller, and K. R. Tanabe, "Treatment of intrahepatic malignancy with radiofrequency ablation," Cancer, vol. 88, no. 11, pp. 2452-2463, 2000.

[6] C. Correa-Gallego, Y. Fong, M. Gonen et al., "A retrospective comparison of microwave Ablation vs. radiofrequency ablation for colorectal cancer hepatic metastases," Annals of Surgical Oncology, vol. 21, no. 13, pp. 4278-4283, 2014.

[7] S. Rossi, F. Fornari, C. Pathies, and L. Buscarini, "Thermal lesions induced by $480 \mathrm{KHz}$ localized current field in guinea pig and pig liver," Tumori, vol. 76, no. 1, pp. 54-57, 1990.

[8] S. Rossi, "Percutaneous ultrasound-guided radiofrequency electrocautery for the treatment of small hepatocellular carcinoma," Journal of Vascular and Interventional Radiology, vol. 8, pp. 97-103, 1993. 
[9] S. A. Sapareto and W. C. Dewey, "Thermal dose determination in cancer therapy," International Journal of Radiation Oncology, Biology \& Physics, vol. 10, no. 6, pp. 787-800, 1984.

[10] D. Haemmerich and D. J. Schutt, "RF ablation at low frequencies for targeted tumor heating: in vitro and computational modeling results," IEEE Transactions on Biomedical Engineering, vol. 58, no. 2, pp. 404-410, 2011.

[11] I. dos Santos, D. Correia, A. J. M. Soares et al., "A surgical device for radiofrequency ablation of large liver tumors," Physiological Measurement, vol. 29, no. 10, pp. N59-N70, 2008.

[12] D. Haemmerich, S. T. Staelin, S. Tungjitkusolmun, F. T. Lee Jr., D. M. Mahvi, and J. G. Webster, "Hepatic bipolar radiofrequency ablation between separated multiprong electrodes," IEEE Transactions on Biomedical Engineering, vol. 48, no. 10, pp. 1145-1152, 2001.

[13] S. Padma, J. B. Martinie, and D. A. Iannitti, "Liver tumor ablation: percutaneous and open approaches," Journal of Surgical Oncology, vol. 100, no. 8, pp. 619-634, 2009.

[14] S. A. Curley, "Radiofrequency ablation of malignant liver tumors," Annals of Surgical Oncology, vol. 10, no. 4, pp. 338-347, 2003.

[15] M. J. Dodd, "Radiofrequency ablation of the liver: current status," American Journal of Roentgenology, vol. 176, pp. 3-16, 2001.

[16] P. L. Pereira, "Actual role of radiofrequency ablation of liver metastases," European Radiology, vol. 17, no. 8, pp. 2062-2070, 2007.

[17] D. Tosi, E. G. Macchi, M. Gallati et al., "Fiber-optic chirped FBG for distributed thermal monitoring of ex-vivo radiofrequency ablation of liver," Biomedical Optics Express, vol. 5, no. 6, pp. 1799-1811, 2014.

[18] D. Tosi, E. G. Macchi, G. Braschi et al., "Fiber-optic combined FPI/FBG sensors for monitoring of radiofrequency thermal ablation of liver tumors: ex vivo experiments," Applied Optics, vol. 53, no. 10, pp. 2136-2144, 2014.

[19] S. Poeggel, D. Tosi, G. Leen, and E. Lewis, "Diaphragm etching in extrinsic Fabry-Perot interferometric fiber optic pressure sensors," in Proceedings of the Conference on and International Quantum Electronics Conference Lasers and ElectroOptics Europe (CLEO '13), Munich, Germany, May 2013.

[20] S. Poeggel, D. Duraibabu, D. Tosi et al., "Novel FBG femtosecond laser inscription method for improved FPI sensors for medical applications," in Proceedings of the IEEE Sensors, pp. 2528, Valencia, Spain, November 2014.

[21] Angiodynamics, Starburst MRI RFA Device.

[22] FP7-STREP IMPPACT, http://www.imppact.eu.

[23] T. Varghese, J. A. Zagzebski, and F. T. Lee, "Elastographic imaging of thermal lesions in the liver in vivo following radiofrequency ablation: preliminary results," Ultrasound in Medicine and Biology, vol. 28, no. 11, pp. 1467-1473, 2002.

[24] K. Ogan, W. W. Roberts, D. M. Wilhelm et al., "Infrared thermography and thermocouple mapping of radiofrequency renal ablation to assess treatment adequacy and ablation margins," Urology, vol. 62, no. 1, pp. 146-151, 2003.

[25] E. Udd and W. B. Spillman, Fiber Optic Sensors: An Introduction for Engineers and Scientists, Wiley, 2nd edition, 2011.

[26] D. Tosi, E. G. Macchi, M. Gallati et al., "Monitoring of radiofrequency thermal ablation in liver tissue through fibre Bragg grating sensors array," Electronics Letters, vol. 50, no. 14, pp. 981983,2014 .
[27] P. Saccomandi, E. Schena, and S. Silvestri, "Techniques for temperature monitoring during laser-induced thermotherapy: an overview," International Journal of Hyperthermia, vol. 29, no. 7, pp. 609-619, 2013.

[28] F. Taffoni, D. Formica, P. Saccomandi, G. di Pino, and E. Schena, "Optical fiber-based MR-compatible sensors for medical applications: an overview," Sensors, vol. 13, no. 10, pp. 14105-14120, 2013.

[29] K. Kotoh, M. Nakamuta, S. Morizono et al., "A multi-step, incremental expansion method for radio frequency ablation: optimization of the procedure to prevent increases in intratumor pressure and to reduce the ablation time," Liver International, vol. 25, no. 3, pp. 542-547, 2005.

[30] P. Roriz, O. Frazão, A. B. Lobo-Ribeiro, J. L. Santos, and J. A. Simões, "Review of fiber-optic pressure sensors for biomedical and biomechanical applications," Journal of Biomedical Optics, vol. 18, no. 5, Article ID 050903, 2013.

[31] G. F. van Hare, M. D. Lesh, and P. Stanger, "Radiofrequency catheter ablation of supraventricular arrhythmias in patients with congenital heart disease: results and technical considerations," Journal of the American College of Cardiology, vol. 22, no. 3, pp. 883-890, 1993.

[32] A. Othonos and K. Kalli, Fiber Bragg Gratings: Fundamentals and Applications, Artech House, 1999.

[33] Y.-J. Rao, "In-fibre Bragg grating sensors," Measurement Science and Technology, vol. 8, no. 4, pp. 355-375, 1997.

[34] T. Erdogan, "Fiber grating spectra," Journal of Lightwave Technology, vol. 15, no. 8, pp. 1277-1294, 1997.

[35] D. J. Webb, M. W. Hathaway, D. A. Jackson, S. Jones, L. Zhang, and I. Bennion, "First in-vivo trials of a fiber Bragg grating based temperature profiling system," Journal of Biomedical Optics, vol. 5, no. 1, pp. 45-50, 2000.

[36] E. Lindner, A. Hartung, D. Hoh et al., "Trends and future of fiber Bragg grating sensing technologies: tailored draw tower gratings (DTGs)," in Optical Sensing and Detection III, 91410X, vol. 9141 of Proceedings of SPIE, Brussels, Belgium, April 2014.

[37] W. Ecke and M. W. Schmitt, "Fiber Bragg gratings in industrial sensing," in Proceedings of the Optical Fiber Communication Conference, Anaheim, Calif, USA, March 2013.

[38] J. M. Gong, C. C. Chan, W. Jin, J. M. K. MacAlpine, M. Zhang, and Y. B. Liao, "Enhancement of wavelength detection accuracy in fiber Bragg grating sensors by using a spectrum correlation technique," Optics Communications, vol. 212, no. 1-3, pp. 29-33, 2002.

[39] H. Shahoei, M. Li, and J. Yao, "Continuously tunable time delay using an optically pumped linear chirped fiber bragg grating," Journal of Lightwave Technology, vol. 29, no. 10, Article ID 5739502, pp. 1465-1472, 2011.

[40] G. Rodriguez, R. L. Sandberg, Q. McCulloch, S. I. Jackson, S. W. Vincent, and E. Udd, "Chirped fiber Bragg grating detonation velocity sensing," Review of Scientific Instruments, vol. 84, no. 1, Article ID 015003, 2013.

[41] Y. Okabe, R. Tsuji, and N. Takeda, "Application of chirped fiber Bragg grating sensors for identification of crack locations in composites," Composites Part A: Applied Science and Manufacturing, vol. 35, no. 1, pp. 59-65, 2004.

[42] J. Skaar, L. Wang, and T. Erdogan, "On the synthesis of fiber Bragg gratings by layer peeling," IEEE Journal of Quantum Electronics, vol. 37, no. 2, pp. 165-173, 2001.

[43] J. Gan, Y. Hao, Q. Ye et al., "High spatial resolution distributed strain sensor based on linear chirped fiber Bragg grating and 
fiber loop ringdown spectroscopy," Optics Letters, vol. 36, no. 6, pp. 879-881, 2011.

[44] J. Skaar and K. M. Risvik, "A genetic algorithm for the inverse problem in synthesis of fiber gratings," Journal of Lightwave Technology, vol. 16, no. 10, pp. 1928-1932, 1998.

[45] E. G. Macchi, M. Gallati, G. Braschi, A. Cigada, and L. Comolli, "Temperature distribution during RF ablation on ex vivo liver tissue: IR measurements and simulations," Heat and Mass Transfer, 2014.

[46] X. Bao and L. Chen, "Recent progress in distributed fiber optic sensors," Sensors, vol. 12, no. 7, pp. 8601-8639, 2012.

[47] K. Hotate and M. Tanaka, "Distributed fiber brillouin strain sensing with $1-\mathrm{cm}$ spatial resolution by correlation-based continuous-wave technique," IEEE Photonics Technology Letters, vol. 14, no. 2, pp. 179-181, 2002.

[48] Y. Lu, T. Zhu, L. Chen, and X. Bao, "Distributed vibration sensor based on coherent detection of phase-OTDR," Journal of Lightwave Technology, vol. 28, no. 22, Article ID 5585644, pp. 3243-3249, 2010.

[49] M. K. Saxena, S. D. V. S. J. Raju, R. Arya, S. V. G. Ravindranath, S. Kher, and S. M. Oak, "Optical fiber distributed temperature sensor using short term Fourier transform based simplified signal processing of Raman signals," Measurement, vol. 47, no. 1, pp. 345-355, 2014.

[50] A. Galtarossa and L. Palmieri, "Spatially resolved PMD measurements," Journal of Lightwave Technology, vol. 22, no. 4, pp. 1103-1115, 2004.

[51] B. J. Soller, D. K. Gifford, M. S. Wolfe, and M. E. Froggatt, "High resolution optical frequency domain reflectometry for characterization of components and assemblies," Optics Express, vol. 13, no. 2, pp. 666-674, 2005.

[52] M. E. Froggatt, D. K. Gifford, S. Kreger, M. Wolfe, and B. J. Soller, "Characterization of polarization-maintaining fiber using highsensitivity optical-frequency-domain reflectometry," Journal of Lightwave Technology, vol. 24, no. 11, pp. 4149-4154, 2006.

[53] D. K. Gifford, B. J. Soller, M. S. Wolfe, and M. E. Froggatt, "Distributed fiber-optic temperature sensing using Rayleigh backscatter," in Proceedings of the 31st European Conference on Optical Communication (ECOC '05), vol. 3, pp. 511-512, September 2005.

[54] D.-P. Zhou, Z. Qin, W. Li, L. Chen, and X. Bao, "Distributed vibration sensing with time-resolved optical frequency-domain reflectometry," Optics Express, vol. 20, no. 12, pp. 13138-13145, 2012.

[55] L. Buscarini, E. Buscarini, M. di Stasi, D. Vallisa, P. Quaretti, and A. Rocca, "Percutaneous radiofrequency ablation of small hepatocellular carcinoma: long-term results," European Radiology, vol. 11, no. 6, pp. 914-921, 2001.

[56] T. Tanaka, N. Yamanaka, T. Oriyama, K. Furukawa, and E. Okamoto, "Factors regulating tumor pressure in hepatocellular carcinoma and implications for tumor spread," Hepatology, vol. 26, no. 2, pp. 283-287, 1997.

[57] M. Nakamuta, M. Kohjima, S. Morizono et al., "Comparison of tissue pressure and ablation time between the LeVeen and cooltip needle methods," Comparative Hepatology, vol. 5, article 10, 2006.

[58] Boston Scientific, LeVeen Needle Electrodes.

[59] Blue Book Memorandum \#G95-1, "Use of International Standard ISO 10993, 'Biological Evaluation of Medical Devices Part 1: Evaluation and Testing,' 1995.
[60] Y.-J. Rao, "Recent progress in fiber-optic extrinsic Fabry-Perot interferometric sensors," Optical Fiber Technology, vol. 12, no. 3, pp. 227-237, 2006.

[61] K. Bremer, E. Lewis, G. Leen, B. Moss, S. Lochmann, and I. A. R. Mueller, "Feedback stabilized interrogation technique for EFPI/FBG hybrid fiber-optic pressure and temperature sensors," IEEE Sensors Journal, vol. 12, no. 1, pp. 133-138, 2012.

[62] P. Polygerinos, D. Zbyszewski, T. Schaeffter, R. Razavi, L. D. Seneviratne, and K. Althoefer, "MRI-compatible fiber-optic force sensors for catheterization procedures," IEEE Sensors Journal, vol. 10, no. 10, pp. 1598-1608, 2010.

[63] H. Bae and M. Yu, "Miniature Fabry-Perot pressure sensor created by using UV-molding process with an optical fiber based mold," Optics Express, vol. 20, no. 13, pp. 14573-14583, 2012.

[64] S. Poeggel, D. Tosi, F. Fusco et al., "Fiber-optic EFPI pressure sensors for in-vivo urodynamic analysis," IEEE Sensors Journal, vol. 14, no. 7, pp. 2335-2340, 2014.

[65] M.-D. Zhou, C. Yang, Z. Liu, J. P. Cysyk, and S.-Y. Zheng, "An implantable Fabry-Pérot pressure sensor fabricated on left ventricular assist device for heart failure," Biomedical Microdevices, vol. 14, no. 1, pp. 235-245, 2012.

[66] C. Liao, S. Liu, L. Xu et al., "Sub-micron silica diaphragmbased fiber-tip Fabry-Perot interferometer for pressure measurement," Optics Letters, vol. 39, no. 10, pp. 2827-2830, 2014.

[67] F. Xu, D. Ren, X. Shi et al., "High-sensitivity Fabry-Perot interferometric pressure sensor based on a nanothick silver diaphragm," Optics Letters, vol. 37, no. 2, pp. 133-135, 2012.

[68] L. H. Chen, C. C. Chan, W. Yuan, S. K. Goh, and J. Sun, "High performance chitosan diaphragm-based fiber-optic acoustic sensor," Sensors and Actuators, A: Physical, vol. 163, no. 1, pp. 42-47, 2010.

[69] K. Bremer, E. Lewis, B. Moss, G. Leen, S. Lochmann, and I. Mueller, "Conception and preliminary evaluation of an optical fibre sensor for simultaneous measurement of pressure and temperature," Journal of Physics: Conference Series, vol. 178, no. 1, Article ID 012016, 2009.

[70] H. Bae, L. Dunlap, J. Wong, and M. Yu, "Miniature temperature compensated Fabry-Perot pressure sensors created with self-aligned polymer photolithography process," IEEE Sensors Journal, vol. 12, no. 5, pp. 1566-1573, 2012.

[71] K. O. Hill, B. Malo, F. Bilodeau, D. C. Johnson, and J. Albert, "Bragg gratings fabricated in monomode photosensitive optical fiber by UV exposure through a phase mask," Applied Physics Letters, vol. 62, no. 10, pp. 1035-1037, 1993.

[72] T. Geernaert, K. Kalli, C. Koutsides et al., "Point-by-point fiber bragg grating inscription in free-standing step-index and photonic crystal fibers using near-IR femtosecond laser," Optics Letters, vol. 35, no. 10, pp. 1647-1649, 2010.

[73] L. Thévenaz, S. Chin, J. Sancho, and S. Sales, "Novel technique for distributed fibre sensing based on faint long gratings (FLOGs)," in 23rd International Conference on Optical Fibre Sensors, vol. 9157 of Proceedings of SPIE, June 2014.

[74] N. Liu, Y. Li, H. Wang, W. Chen, and P. Lu, "Directional bend sensing with bragg gratings in all solid bragg fibers," IEEE Photonics Technology Letters, vol. 23, no. 17, pp. 1237-1239, 2011.

[75] D. Tosi, S. Poeggel, G. Leen, and E. Lewis, "Adaptive filterbased interrogation of high-sensitivity fiber optic Fabry-Perot interferometry sensors," Sensors and Actuators, A: Physical, vol. 206, pp. 144-150, 2014. 
[76] X. Liu, I. I. Iordachita, X. He, R. H. Taylor, and J. U. King, "Miniature fiber-optic force sensor based on low-coherence Fabry-pérot interferometry for vitreoretinal microsurgery," Biomedical Optics Express, vol. 3, no. 5, pp. 1062-1076, 2012.

[77] A. R. Lanfranco, A. E. Castellanos, J. P. Desai, and W. C. Meyers, "Robotic surgery: a current perspective," Annals of Surgery, vol. 239, no. 1, pp. 14-21, 2004.

[78] R. C. G. Martin, C. R. Scoggins, and K. M. McMasters, "Safety and efficacy of microwave ablation of hepatic tumors: a prospective review of a 5-year experience," Annals of Surgical Oncology, vol. 17, no. 1, pp. 171-178, 2010.

[79] S. Mulier, Y. Jiang, C. Wang et al., "Bipolar radiofrequency ablation with four electrodes: ex vivo liver experiments and finite element method analysis. Influence of inter-electrode distance on coagulation size and geometry," International Journal of Hyperthermia, vol. 28, no. 7, pp. 686-697, 2012.

[80] W. B. Shingleton and P. E. Sewell Jr., "Percutaneous renal tumor cryoablation with magnetic resonance imaging guidance," The Journal of Urology, vol. 165, no. 3, pp. 773-776, 2001. 

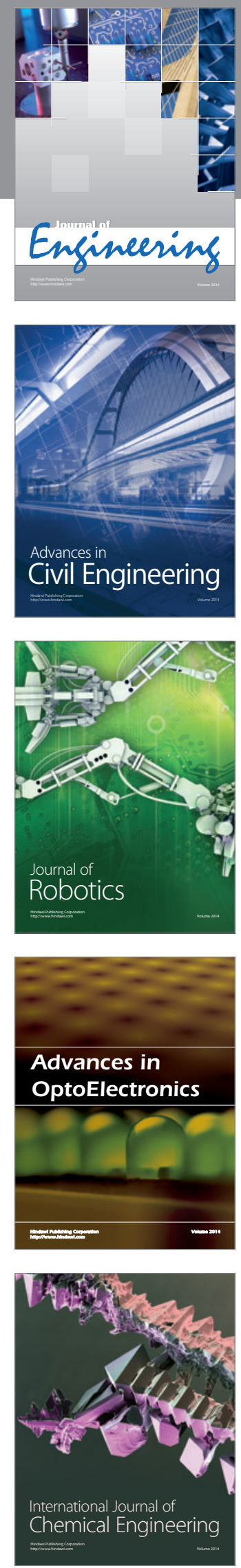

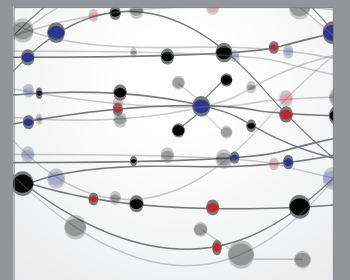

The Scientific World Journal
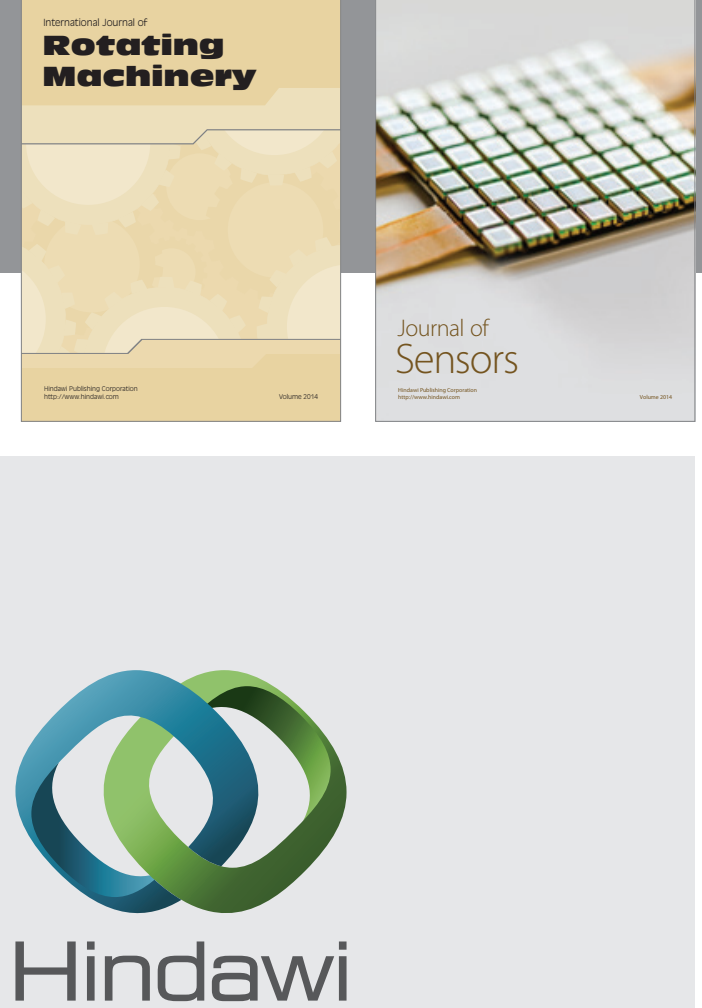

Submit your manuscripts at http://www.hindawi.com
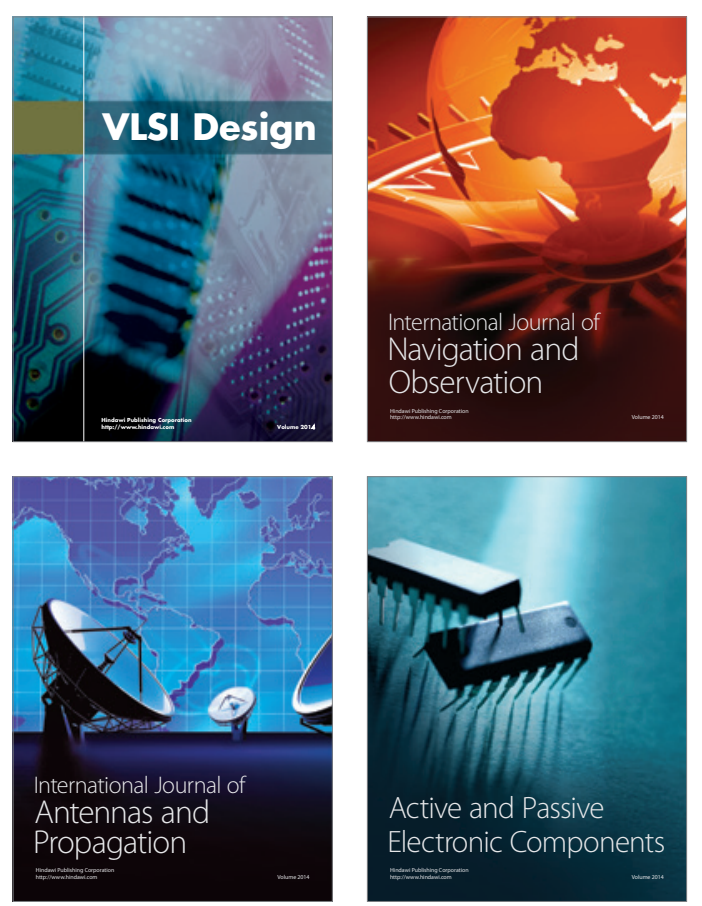
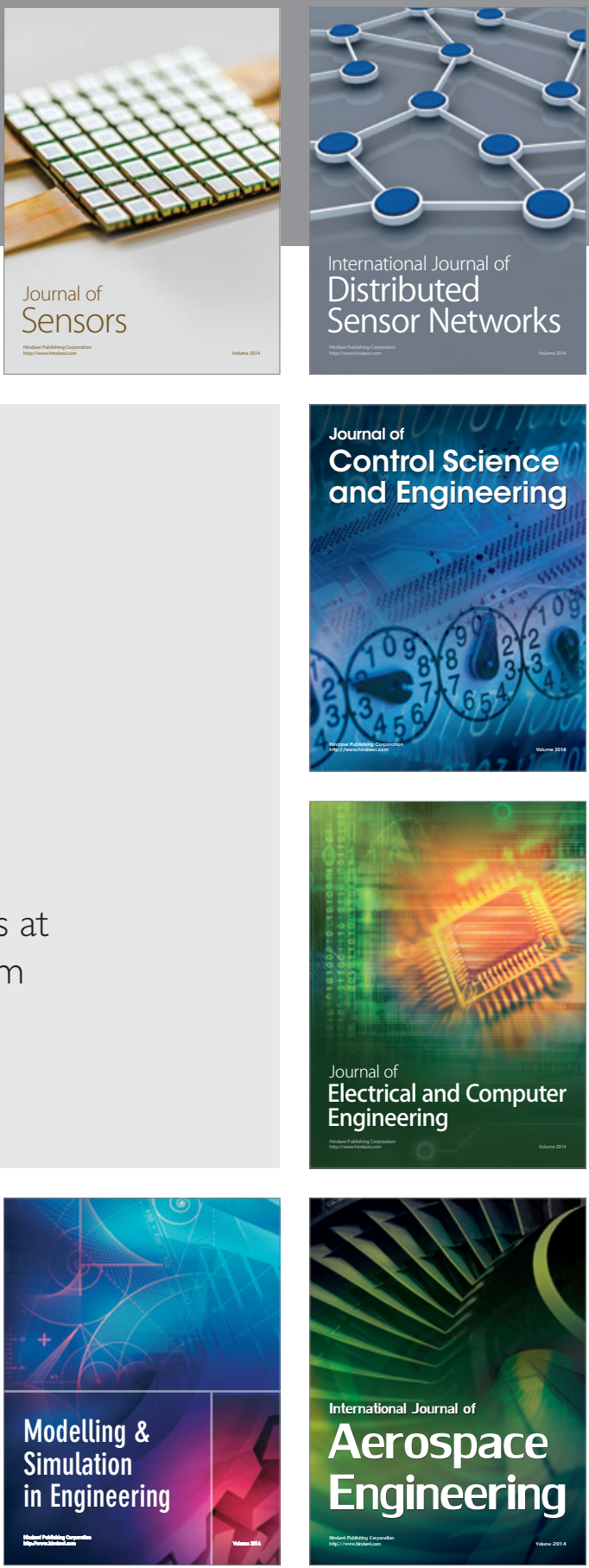

Journal of

Control Science

and Engineering
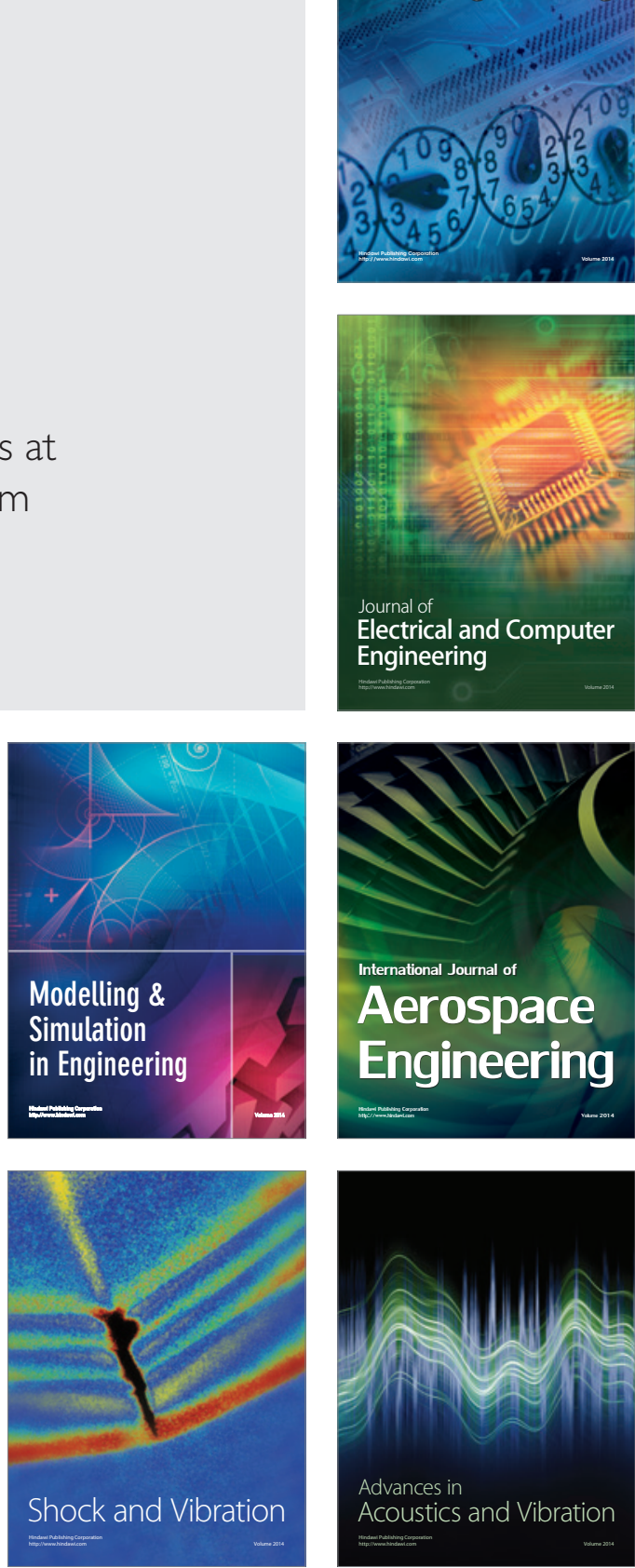